\title{
Sociedades cooperativas de trabajo asociado versus sociedades de capital. Análisis comparado de fortaleza financiera y rentabilidad en el País Vasco
}

\section{Miguel Ángel Zubiaurre, Lorea Andicoechea y Ainhoa Saitua}

\section{RESUMEN}

Tradicionalmente, los agentes económicos de la economía social y, en particular las cooperativas, se habían mostrado más resistente en entornos de crisis, generando mejores resultados en términos de mantenimiento de empleo. El estallido del caso Fagor Electrodomésticos ha supuesto un estímulo que ha extendido la necesidad de revisar algunos de los parámetros del modelo cooperativo.

En este sentido, el trabajo realiza un análisis comparado de la estructura financiera, la solvencia, la rentabilidad y la capacidad de generar riqueza de las sociedades cooperativas respecto a las sociedades de capital. Según los resultados del trabajo entre las empresas industriales de más de 50 empleados del País Vasco, las cooperativas ofrecen indicadores similares o superiores a las sociedades anónimas y limitadas. Por tanto, el cooperativismo industrial vasco presenta datos positivos que muestran su capacidad de mantenerse como una fuente de generación de riqueza y empleo.

PALABRAS CLAVE: Cooperativa, Sociedad de Capital, Rentabilidad, Solvencia, Análisis.

CLAVES ECONLIT: A13, M20, G32, P13.

Cómo citar este artículo: ZUBIARRE, M.A., ANDICOECHEA, L. \& SAITUA, A. (2016): "Sociedades cooperativas de trabajo asociado versus sociedades de capital. Análisis comparado de fortaleza financiera y rentabilidad en el País Vasco", CIRIEC-España, Revista de Economía Pública, Social y Cooperativa, 86, 155-194.

Correspondencia: Miguel Ángel Zubiaurre (correspondencia), Profesor Titular de la Universidad del País Vasco (UPV/EHU); Lorea Andicoechea, Profesora Titular de la Universidad del País Vasco (UPV/EHU), y Ainhoa Saitua, Profesora Doctora Titular de Escuela Universitaria (UPV/EHU).

E-mail: ma.zubiaurre@ehu.es 


\section{EXPANDED ABSTRACT}

\section{Worker cooperatives versus investor-owned firms. Comparative analysis of financial strength and performance in the Basque Country}

\section{Objective}

Traditionally, agents of social economy and, particularly cooperatives, have been more resilient in crisis environments, thus generating better results in terms of job retention. However, the bankruptcy of Fagor Electrodomésticos, a very large cooperative in the Basque Country (Spain), has given rise to review some of the elements of the cooperative model.

The aim of this paper is to make an assessment of the current financial position of cooperative enterprises in the Basque Country, by proposing a comparative analysis of the financing structure, solvency and profitability of cooperatives with regards to investor owned firms.

This objective is completed through the following steps:

- By identifying what the current financial structure, solvency and profitability of the Basque cooperatives is, through a thorough analysis of their annual accounts.

- By conducting an exploratory comparative assessment of the financial structure, solvency and profitability of cooperative entities regarding investor owned firms.

- By checking whether there are significant differences between the cooperative and capitalist models in their financial structures in terms of rates of return and wealth generation.

In fact, the interest in comparing the two business models lies in the specific principles and values the cooperative model assumes, which comprises a more democratic and participatory system of government. Besides, it is important to notice that in our study we deal with worker cooperatives, which involves unifying the position of equity contributors and workers.

There are other peculiarities in self-financing policies and surplus distribution. The different regulatory framework forces cooperatives to allocate larger percentages to legal reserves. On the other hand, it is common that assemblies of members choose to capitalize returns rather than pay out them. Consequently, the recovery of the returns generated is delayed until partners' departure or its retirement time. These policies are aimed at strengthening their own resources as well as improving the solvency ratio of cooperatives. Another specific important policy is related to the member's capital contribution. 
In essence, the data obtained will allow us to conclude whether the singularities of the cooperative model generate significant differences on their financial structures compared to investor owned firms. In addition, we will research if there are significant differences in their profitability indicators and ability to generate wealth.

\section{Methodology}

We are presenting an exploratory work of the economic and financial position of cooperatives concerning capital companies. The study has been conducted by analysing data presented in the annual financial statements of industrial cooperatives with more than 50 employees located in the Basque Country, during the period of 2010-2011. We have therefore evaluated, the financial statements of 50 of the 65 cooperatives that met these conditions, in total representing $77 \%$ of the study population. In order to selectothe corporate enterprises, we have identified companies with more than 50 workers located in the Basque Country operating in any of the industries in which sample cooperatives work. Additionally, 27 economic-financial indicators have been considered to perform a comparative analysis for each year (2010 and 2011) therefore representing a total of 54 variables, for each type of entity. We have identified nine indicators to assess the solvency and financial structure on three aspects: liquidity, global solvency and financial ability to repay debts. We have identified 18 indicators on three aspects to analyze the profitability and wealth generation capability: profitability returns, value added and productivity. The singularities of the worker cooperative model, where labor and capital have concurring interests, require alternative instruments to the classic profitability ratios, so as to properly compare the wealth generating capability of both models.

\section{Results}

The results show that the studied worker cooperatives offer similar or superior indicators to investor owned firms. Cooperatives have a lower level of debt and thus a higher level of solvency, in line with results obtained by Chaddad (2001), Soboh et al. (2012) and Pozuelo et al. (2012). This suggests that policies to strengthen the legally imposed self-financing are able to counteract the traditional limitation when attracting equity capital, resulting in a more solid financial structure in cooperatives.

In addition, we have made progress identifying the reasons for the observed different levels of indebtedness, which are related to a lower level of short term debts. In our opinion, the identified differences could be explained by a higher level of commercial debt in corporate enterprises. In any case, the results are inconclusive in identifying the financial debt as a factor to explain the lower level of overall indebtedness observed in cooperatives.

Moreover, achieving adequate rates of return is one of the basic elements required for the survival of any business. The results of the analysis, although not statistically significant, suggest higher rates of economic and financial profitability in cooperatives, in this way contradicting most previous works, although being consistent with those observed by Hind (1994). 
Significant differences were also observed in productivity indicators, which show a greater capacity to generate value over investment in personnel in cooperatives. Additionally, the operating profit per employee is higher in cooperatives than in investor owned firms.

Although throughout crisis times cooperatives are associated to better job retention levels, this policy seems to have no impact on the return rates observed in our study. This could be explained by the wage adjustments seen in worker cooperatives, so as to prevent redundancies of members.

\section{Limitations}

The project only considers two-year worth of data, so we have not enough evidence to draw any relevant conclusions on the evolution of the observed indicators. Future work will study the effects of the global crisis on the two groups using longer time series. It will also be of interest to go deeper into the individualized analysis of the specific characteristics and policies of cooperatives as a cause of the identified differences.

\section{Conclusions}

Despite the convulsion that the bankruptcy of Fagor Electrodomésticos - one of the leading cooperatives- caused, the Basque cooperativism presents better indicators in most of the variables compared to those of capital companies, which allows us to consider them as a fundamental driver for of wealth and employment in the Basque Country.

The results may be useful when considering the revision and consolidation of some of the elements that have shaped the cooperative model up to the present, with the aim of contributing to the consolidation and strength of cooperatives as alternatives to generate wealth and employment.

KEY WORDS: Cooperatives, Investor-owned Firms, Profitability, Solvency, Analysis. 


\section{1.- Introducción}

El actual contexto internacional de creciente globalización y complejidad, de pérdida de peso de la economía productiva respecto a la economía financiera, está generando consecuencias negativas en términos de pérdidas de empleo y retrocesos en el estado de bienestar en las economías más desarrolladas. La Comisión Europea planteó en el 2010 la Estrategia de la Unión Europea 2020, a fin de lograr una recuperación sostenible, cuya finalidad no es sólo superar la crisis que continúa azotando a muchas de sus economías, sino también subsanar los defectos del modelo de crecimiento y crear las condiciones propicias para un tipo de crecimiento distinto, más inteligente, sostenible e integrador. Entre los cinco objetivos específicos fijados se contempla la creación de empleo y la lucha contra la pobreza y la exclusión social (Comisión Europea, 2010). Se constata que los indicadores de tasa de empleo empeoran a nivel global, por lo que el objetivo pretendido en el 2020 de una tasa de empleo del 75\% de los habitantes entre 20-64 años no parece fácilmente alcanzable. Lógicamente, el informe de la comisión menciona a España entre los países en los que la consecución del objetivo no parece factible. En este entorno de crisis y de destrucción de empleo, la "economía social" parece estar llamada a cumplir un papel relevante, tal y como señalan diferentes trabajos (Stiglitz, 2009; Birchall y Ketilson, 2009; Cooperatives Europe, 2010; Mckinsey, 2012; Monzón y Chaves, 2012).

Según los datos ofrecidos por el informe sobre la economía social en Europa (Monzón y Chaves, 2012), el peso relativo de la economía social en el total de los empleos de Unión Europea ha crecido durante los últimos años suponiendo en 2009-2010 un 6,5\% del empleo total de UE. Estos datos son extrapolables a España, donde el conjunto de la economía social suponía el 6,74\% del empleo de total del país. Un hecho singular del caso español es que el $52 \%$ de los empleos que genera la economía social corresponden al modelo cooperativo, lo que supone veinte puntos porcentuales más que la media de los países de la UE, alcanzando el 3,5\% del empleo total del país. En la Comunidad Autónoma del País Vasco (CAPV) este porcentaje es aún superior y en el 2011, según datos del Observatorio Vasco de Economía Social (2012), el empleo ofrecido por empresas cooperativas suponía el 5,07\% del empleo total de la CAPV. Es además destacable, que el 9,29\% de la actividad industrial del CAPV se ejerce a través de las cooperativas. Esta singularidad supone un elemento diferenciador de nuestro entramado social y económico, muy reconocido a nivel internacional que ha contribuido de manera muy significativa a la generación de empleo y riqueza en el CAPV.

Con estos antecedentes, el objetivo del trabajo consiste en realizar una valoración de la situación actual de las empresas cooperativas del País Vasco, planteando un análisis comparado de la estructura de financiación, de la solvencia y de la rentabilidad de las sociedades cooperativas respecto a las sociedades de capital. El estudio se realizará a partir de la información financiera que presen- 
tan las cuentas anuales de las empresas industriales de más de 50 trabajadores con sede social en el País Vasco durante el periodo 2010-2011. Los datos obtenidos nos permitirán concluir si las singularidades del modelo cooperativo respecto a las sociedades de capital, en cuanto a mayores restricciones en el reparto de excedente, el sistema de aportación y rescate del capital, o los compromisos de remuneración de capital pactados en estatutos, generan diferencias significativas sobre sus estructuras financieras. Además, se diagnosticará si existen diferencias significativas en cuanto a tasas de rentabilidad y capacidad de generar riqueza. Los resultados obtenidos pueden ser de utilidad a la hora de plantear la revisión de algunos de los elementos que han configurado el modelo cooperativo hasta el presente con el objetivo de contribuir a consolidar y fortalecer las cooperativas como alternativas de generación de riqueza y empleo.

A continuación el apartado segundo revisa el marco teórico que apoya el estudio. El tercero explica la metodología utilizada y el cuarto comenta los resultados obtenidos. En el último, se presentan las reflexiones finales y las posibles líneas de investigación futuras.

\section{2.- Marco teórico}

Las cooperativas están caracterizadas por modelos de gobernanza más democráticos y participativos en los que el trabajo y la aportación de capital comparten compromisos y objetivos comunes. Esta circunstancia genera singularidades en el modo en el que se configuran los capitales propios y se reparten los excedentes empresariales (García-Gutiérrez, 1986; Bel y Fernández, 2002; Zubiaurre, 2004; Gómez y Miranda, 2006; AECA, 2009 y 2010). La legislación de cooperativas en España, si bien existen singularidades en las normativas autonómicas en relación a la disponibilidad (ver AECA, 2009), contempla mayores exigencias de dotación de reservas en el reparto de los excedentes anuales. En general, las cooperativas se ven obligadas a destinar porcentajes superiores a las sociedades de capital a fondos de reserva obligatorios y, además, lo han de hacer de modo continuado a lo largo de su vida económica sin quedar supeditados a la consecución de objetivo basado en un porcentaje del capital, como es el caso de las sociedades anónimas y limitadas. Por otro lado, es común que las asambleas de las cooperativas opten por capitalizar los retornos en lugar de monetizarlos. De este modo, la recuperación de buena parte de los rendimientos generados a lo largo de la vida de laboral, se retrasa hasta el momento de la jubilación o abandono de la cooperativa por parte de los socios. Estas políticas están destinadas al fortalecimiento de los recursos propios y, por tanto, también de la solvencia de las sociedades cooperativas. 
Existen, sin embargo, corrientes de pensamiento que argumentan que la escasez de financiación vía capital es un factor explicativo de la todavía limitada incidencia del modelo cooperativo en la economía mundial. La inexistencia de una relación de propiedad sobre los activos de las cooperativas por parte de los socios se identifica como un elemento desincentivador a la retención de resultados en los fondos propios (Furubotn y Pejovich, 1970; Vanek, 1977; Bonin, Jones y Putterman, 1993). En todo caso, la coexistencia de los intereses particulares de los socios y de los intereses de la cooperativa en el momento de la toma de decisión sobre el reparto de los excedentes genera conflictos de interés.

A pesar de estas argumentaciones teóricas, existen estudios (Ben-Ner, 1988; Estrin y Jones, 1992; Zevi, Zanotti, Soulage y Zelaia, 2011; Cantarero González-Loureiro y Puig, 2013) realizados sobre diferentes países europeos que constatan cómo las cooperativas han sido capaces de evitar mejor los efectos de la crisis y ofrecer mejores tasas de supervivencia. También en España, Díaz y Marcuello (2010), Calderón y Calderón (2012) y Server y Lajara-Camilleri (2012) constatan mejores datos en términos de mantenimiento del empleo bajo el modelo cooperativo en periodos de recesión.

En la Comunidad Autónoma del País Vasco, la caída de Fagor Electrodomésticos, así como las dificultades de refinanciación de deuda de Eroski, han supuesto un estímulo que ha extendido la idea de la necesidad de revisar algunas de las características esenciales del modelo cooperativo a fin de garantizar una estructura financiera adecuada que permita afrontar con éxito los retos de una economía globalizada.

En la literatura previa existen diversos trabajos empíricos que tienen como objetivo realizar un análisis comparado sobre las diferencias en indicadores de solvencia, rentabilidad o productividad entre las cooperativas y las empresas de índole puramente capitalista. Estos estudios no llegan a conclusiones uniformes. En Estados Unidos Chaddad (2001) constataba que las políticas de inversión de las cooperativas agrícolas restringían las posibilidades de autofinanciación, lo cual reducía los niveles de endeudamiento. De modo similar, Soboh, Oude-Lansink y Van Dijk (2011) pudieron constatar que las cooperativas ofrecían un nivel de endeudamiento menor y, por tanto, una mejor posición financiera que las empresas capitalistas en el sector lácteo europeo. Por el contrario, Notta y Vlachvei (2007) obtuvieron resultados que indicaban que las cooperativas presentan un mayor nivel de endeudamiento que las empresas capitalistas en su estudio en el sector lácteo.

Por otra parte, en el trabajo de Bartlett, Cable, Estrin, Jones y Smith (1992) para empresas italianas, a pesar de identificar valores medios inferiores en el porcentaje de financiación propia de las cooperativas, las diferencias no resultaron estadísticamente significativas. Tampoco encuentran diferencias significativas en cuanto a posición financiera entre cooperativas y empresas de capital Syrjä, Sjogrén y Touminen (2012) en las cooperativas finlandesas de consumo analizadas.

En el caso español, en el estudio comparativo de cooperativas y PYMES de la Comunidad Valenciana de Pozuelo, Carmona y Martínez (2012), los resultados en términos de estructura financiera ofrecen mayores porcentajes de fondos propios en las cooperativas, lo que favorece los ratios 
de solvencia global de las mismas. En cambio, Amat y Perramón (2011) identifican comportamientos diferentes en el modelo de financiación de las cooperativas con altas tasas de crecimiento, respecto de las sociedades de capital. Su estudio constata que las cooperativas han recurrido en mayor proporción a fuentes de financiación ajenas. Este apalancamiento les ha permitido mantener niveles de rentabilidad financiera similares a las empresas capitalistas pero, lógicamente, el riesgo asumido puede condicionar la rentabilidad futura.

\section{Cuadro 1. Revisión de estudios comparativos de cooperativas y sociedades capitalistas sobre endeudamiento}

\begin{tabular}{|c|c|c|c|}
\hline \multirow{2}{*}{ Estudio } & Contexto & \multicolumn{2}{c|}{ Endeudamiento en Cooperativas } \\
\cline { 3 - 4 } & & MENOR & MAYOR \\
\hline Chaddad (2001) & Estados Unidos. Sector agrícola & $\sqrt{ }$ & \\
\hline Soboh, Oude-Lansink y Van Dijk (2011) & Europa. Sector lácteo & $\sqrt{ }$ \\
\hline Notta y Vlachvei (2007) & Grecia. Sector lácteo & & $\sqrt{ }$ \\
\hline Bartlett, Cable, Estrin, Jones y Smith (1992) & Italia (Toscana y Emilia-Romagna) & \multicolumn{2}{|c|}{ No diferencias significativas } \\
\hline Syrjä, Sjogrén y Touminen (2012) & Finlandia. Cooperativas de consumo & No diferencias significativas \\
\hline Pozuelo, Carmona y Martínez (2012) & España, Comunidad Valenciana, PYMES & $\sqrt{ }$ & \\
\hline Amat y Perramón (2011) España & Cooperativas con altas tasas de crecimiento & & $\sqrt{ }$ \\
\hline
\end{tabular}

En los estudios previos, existe mayor consenso sobre los resultados del análisis comparado en términos de rentabilidad. Las investigaciones de Soboh et al. (2011 y 2012), Notta y Vlachvei (2007), Syrjä et al. (2012) y Pozuelo et al. (2012) concluyen que los índices de rentabilidad de las cooperativas son inferiores a los de las sociedades de capital. Sin embargo, también podemos encontrar trabajos que no encuentran diferencias significativas (Hind, 1994).

\section{Cuadro 2. Revisión de estudios comparativos de cooperativas y sociedades capitalistas sobre rentabilidad}

\begin{tabular}{|c|c|c|c|}
\hline Estudio & Contexto & \multicolumn{2}{|c|}{ Rentabilidad en Cooperativas } \\
\cline { 3 - 4 } & & MENOR & MAYOR \\
\hline Soboh et al. (2011, 2012) & Europa. Sector lácteo & $\sqrt{ }$ & \\
\hline Notta y Vlachvei (2007) & Grecia. Sector lácteo & $\sqrt{ }$ & \\
\hline Syrjä et al. (2012) & Finlandia. Cooperativas de consumo & $\sqrt{ }$ & \\
\hline Pozuelo et al. (2012) & España, Comunidad Valenciana, PYMES & $\sqrt{ }$ \\
\hline Hind (1994) & Reino Unido. Sector Agrícola & \multicolumn{2}{|c|}{ No hay diferencias significativas } \\
\hline
\end{tabular}


En todo caso, la búsqueda del objetivo de maximización del beneficio, definido en los términos de la empresa capitalista, no resulta directamente extrapolable a los objetivos de las cooperativas. La singularidad de las conexiones que el socio de la cooperativa puede tener en su doble condición de sociotrabajador, socio-proveedor o socio-consumidor, hace necesario recurrir a parámetros de medición de eficiencia económica alternativos, a fin de realizar un análisis comparado homogéneo que supere las diferentes concepciones de modelos de empresa. En este sentido, Bartlett et al. (1992) utilizan indicadores basados en términos de valor añadido, constatando mejores resultados en las sociedades cooperativas que en las capitalistas.

Por otra parte, es necesario señalar que las entidades cooperativas españolas se han visto sometidas a un nuevo marco normativo contable aprobado por la Orden EHA/3360/2010 que entró en vigor en 2011. Este hecho ha supuesto la aplicación plena de las normas internacionales de contabilidad a las cooperativas, dando por concluido el periodo transitorio abierto tras la aprobación del Plan General de Contabilidad de 2007. Este proceso ha suscitado un amplio debate académico sobre la oportunidad de aplicar a las sociedades cooperativas una normativa internacional desarrollada para su aplicación en el entorno de las sociedades de capital (Fernández-Feijóo y Cabaleiro, 2007; Cubedo, 2007; Polo-Garrido, 2007; Cabaleiro, Ruiz y Fernández-Feijóo, 2011; Andicoechea y Zubiaurre, 2012).

La amenaza más clara de la aplicación de la nueva norma contable consistía en la pérdida de la calificación como patrimonio neto de las aportaciones del capital de los socios de las cooperativas, que ha sido tradicionalmente el componente central del neto patrimonial de las entidades cooperativas. Su reclasificación como pasivo suponía un serio riesgo a la imagen de solvencia de las cuentas anuales de estas entidades (Vargas, 2007; Fernández 2007; Genovart, 2012). Además, el diferenciado desarrollo normativo en materia de cooperativa a nivel autonómico hace que en algunas comunidades exista, a su vez, un hipotético riesgo de disolución (Vargas, 2011).

Ante la previsible asunción de los criterios del regulador internacional de contabilidad en la normativa contable española de cooperativas, y con el objetivo de poder mantener el capital como fondo propio, se procedió a reformar la legislación en materia de cooperativas abriendo la posibilidad de configurar el capital de las cooperativas como aportaciones cuyo reembolso pueda ser rehusado incondicionalmente por el Consejo Rector en caso de baja. En este sentido, la modificación de la Ley de Cooperativas de Euskadi fue una iniciativa pionera a nivel nacional, que posteriormente fue incorporada a la Ley de Cooperativas Estatal y a las diferentes normativas autonómicas ${ }^{1}$. Lógicamente, cada cooperativa deberá tomar en Asamblea la decisión de reconfigurar o no su capital bajo esta nueva opción. Si lo hace así, la cooperativa no tendrá impedimento para mantenerlo como fondo propio. No obstante, tal y como manifiestan Martín, Lejarriaga e Iturrioz (2007), esta iniciativa desvirtúa parte de la esencia de las sociedades cooperativas. 
Los efectos de este cambio normativo han sido estudiados aplicando diversa metodología. Con carácter hipotético, Marí-Vidal (2006) analizó el efecto que podría tener la reclasificación del capital como pasivo sobre diversos ratios financieros. Igualmente, Arias y Montegut (2012) estudiaron el efecto que diversas hipótesis de reclasificación de capital podrían tener sobre los ratios de solvencia y rentabilidad en 15 cooperativas catalanas. Posteriormente, una vez que las empresas han presentado datos financieros del ejercicio 2011, se han realizado estudios basados en datos reales (Bastida y Carreras, 2013; Zubiaurre, Andicoechea y Saitua, 2015) para observar las consecuencias efectivas de su aplicación ofrecen resultados diferentes en las comunidades autónomas analizadas. Zubiaurre et al. (2015) constatan que en la Comunidad Autónoma del País Vasco su aplicación no ha tenido un impacto relevante, fundamentalmente, porque las cooperativas han optado por evitar la consideración de pasivo de las aportaciones cambiando las condiciones estatutarias del capital a fin de protegerse del efecto de la aplicación del nuevo marco normativo contable. Sin embargo, el efecto conjunto de los ajustes se ha mostrado significativo en el estudio realizado sobre cooperativas catalanas (Bastida y Carreras, 2013).

\section{3.- Objetivos de la investigación y metodología empleada}

\subsection{Definición de objetivos}

El objetivo de este trabajo consiste en realizar un análisis comparado de la estructura de financiación, de la solvencia y de la rentabilidad de las sociedades cooperativas con respecto a las sociedades de capital en el País Vasco. En concreto, se persiguen los siguientes objetivos:

- Identificar la situación actual en cuanto a la estructura financiera, solvencia y rentabilidad de las empresas del entorno cooperativo vasco, a través del análisis de la información financiera reflejada en sus cuentas anuales.

- Realizar un análisis exploratorio comparado de la estructura de financiación, de la solvencia y de la rentabilidad de las sociedades cooperativas respecto a las sociedades de capital.

- Verificar si existen diferencias significativas entre el modelo cooperativo y capitalista en cuanto a sus estructuras financieras.

- Verificar si existen diferencias significativas entre el modelo cooperativo y capitalista en cuanto a tasas de rentabilidad y generación de valor. 


\subsection{Selección de la muestra}

Para realizar el análisis se ha seleccionado una muestra representativa de sociedades cooperativas y otra de las sociedades capitalistas que puedan resultar comparables atendiendo a su tamaño y sector de actividad. En el ámbito cooperativo, el estudio se ha centrado en las cooperativas industriales de trabajo asociado con más de 50 trabajadores que tengan domicilio social en la Comunidad autónoma del País Vasco. La identificación de las cooperativas objeto de estudio se realizó a través de los registros disponibles en la Federación de Cooperativas de Trabajo Asociado, Enseñanza y Crédito del País Vasco (ERKIDE). Una vez verificado que 65 cooperativas cumplían las condiciones exigidas, del Registro de Cooperativas del País Vasco se obtuvo copia de las cuentas anuales individuales del ejercicio 2011 de 50 empresas que componen la muestra analizada (suponen el $77 \%$ de la población estudiada), y de las que 47 estaban auditadas. El 78\% de las cooperativas analizadas (39 empresas) pertenecen al grupo Mondragon Corporación Cooperativa (MCC), referente en el movimiento cooperativista en Euskadi desde la década de 1960 (Henk, 1982; White y White, 1991; Ormaechea, 1991; Bakaikoa, Errasti y Begiristain, 2004).

Para la selección de las sociedades anónimas y limitadas se ha utilizado información disponible en la base de datos SABI (Sistemas de Análisis de Balances Ibéricos). Así, se han identificado las sociedades anónimas y limitadas de más de 50 trabajadores que tengan su sede social en el País Vasco, con información disponible para los ejercicios 2010 y 2011, y que operen en alguna de las actividades (códigos CNAE 2009) en las que se había identificado alguna cooperativa industrial. El número de empresas identificado ascendió a 337 sociedades anónimas y limitadas. Al tratarse de empresas industriales de más de 50 trabajadores se genera una homogeneidad en la dimensión y la actividad entre las dos muestras comparadas. En la tabla 1 se describen las actividades industriales de las empresas de la muestra, pudiéndose observar una gran similitud en el peso relativo de los subsectores a nivel de un dígito en cada muestra comparativa. 


\section{Tabla 1. Número de empresas de la muestra por códigos de actividad}

\begin{tabular}{|l|c|c|c|c|c|c|}
\hline CNAE 2009 INDUSTRIA & $\begin{array}{c}\text { Núm. de } \\
\text { Coops. }\end{array}$ & $\begin{array}{c}\% \text { sobre } \\
\text { el sector a } \\
\text { dos dígitos }\end{array}$ & $\begin{array}{c}\% \text { sobre } \\
\text { el sector } \\
\text { a un dígito }\end{array}$ & $\begin{array}{c}\text { Núm. de } \\
\text { SA y SL }\end{array}$ & $\begin{array}{c}\% \text { sobre } \\
\text { el sector a } \\
\text { dos dígitos }\end{array}$ & $\begin{array}{c}\% \text { sobre } \\
\text { el sector } \\
\text { a un dígito }\end{array}$ \\
\hline $\begin{array}{c}16 \text { Industria de la madera y del corcho; excepto } \\
\text { muebles; cestería y espartería }\end{array}$ & 1 & $2,00 \%$ & $2,00 \%$ & 4 & $1,19 \%$ & $1,19 \%$ \\
\hline 20 Industria química & 1 & $2,00 \%$ & $88 \%$ & 16 & $4,75 \%$ & $89 \%$ \\
22 Fabricación de productos de caucho y plásticos & 3 & $6,00 \%$ & 25 & $7,42 \%$ & \\
24 Metalurgia; fabricación de productos de hierro; & 1 & $2,00 \%$ & & 54 & $16,02 \%$ & \\
$\quad$ acero y ferroaleaciones & 12 & $24,00 \%$ & & 89 & $26,41 \%$ & \\
25 Fabricación de productos metálicos; excepto \\
maquinaria y equipo
\end{tabular}

Por último, debemos señalar que en el caso de las cooperativas, las cuentas anuales de fecha 31/12/2011 fueron las primeras en las que resultaba de obligado cumplimiento la aplicación de la nueva norma de contabilidad de cooperativas (Orden EHA/3360/2010). La interpretación de la disposición transitoria única en la que se desarrollaban los criterios para la primera aplicación, ha resultado heterogénea, dificultando la aplicación de nuestro estudio. La mayoría de las cooperativas optó por facilitar la comparabilidad de la información de pérdidas y ganancias presentando los resultados del 2010 bajo la estructura de la cuenta de resultados aprobada por la Orden EHA/3360/2010. No obstante, este esfuerzo de adaptación ha ofrecido resultados poco satisfactorios en lo que respecta a la coherencia de la reclasificación de los intereses retribuidos al capital en el 2010, que en ocasiones eran reclasificados como aplicación del resultado, mientras que en otros casos se seguían presentando como gasto dentro del resultado financiero. La reclasificación de la dotación del fondo de educación como componente del resultado de explotación ha resultado más acertada. Estas circunstancias han exigido un esfuerzo de reelaboración de la información presentada en los estados financieros de las diferentes cooperativas, especialmente al cierre 2010, al objeto de obtener unos resultados homogéneos y comparables. 


\subsection{Definición de indicadores}

A fin de cumplir con los objetivos principales del trabajo, hemos definido los ratios clasificados en dos bloques:

- Análisis de Solvencia y Estructura Financiera.

- Análisis de Rentabilidad y Capacidad de Generar Riqueza.

Por una parte, para la evaluación de la solvencia y la estructura financiera hemos identificado, a su vez, tres subáreas de interés, desplegando un total de 9 indicadores:

a) Solvencia a corto plazo y liquidez.

b) Solvencia global y estructura financiera.

c) Capacidad de devolución de deudas financieras.

En el área de la solvencia a corto plazo se han calculado los tres indicadores clásicos de la solvencia corto plazo, prueba ácida y ratio de disponibilidad o liquidez inmediata.

\section{SOLVENCIA A CORTO PLAZO - LIQUIDEZ}

SOLVCP 1 ACTIVO CORRIENTE / PASIVO CORRIENTE

SOVLCP 2 (ACTIVO CORRIENTE-EXISTENCIAS) / PASIVO CORRIENTE

SOLVCP $3 \quad$ DISPONIBLE / PASIVO CORRIENTE

A fin de evaluar la estructura financiera y la solvencia a largo plazo de las empresas hemos recurrido, además del ratio de solvencia global, a otros tres ratios que buscan evaluar la dependencia financiación externa.

\section{SOLVENCIA A LARGO PLAZO - ESTRUCTURA FINANCIERA}

SOLVLP 1 ACTIVO TOTAL / (PASIVO NO CORRIENTE + PASIVO CORRIENTE)

SOLVLP 2 ACTIVO NO CORRIENTE / PATRIMONIO NETO

SOLVLP $3 \quad$ PATRIMONIO NETO / (PASIVO NO CORRIENTE + PATRIMONIO NETO)

SOLVLP $4 \quad$ DEUDA FINANCIERA / (PASIVO NO CORRIENTE + PASIVO CORRIENTE)

Los ratios de estructura financiera de balance se han completado con dos ratios que evalúan la capacidad de devolución de deudas financieras en función de la generación de recursos (Ebitda) 0 efectivo (flujo de efectivo de las actividades de explotación). La variable Ebitda ha sido determinada a través de la información del estado de flujos de efectivo, quedando definida como el resultado antes de impuestos más los ajustes al resultado.

\section{CAPACIDAD DE DEVOLUCIÓN DE DEUDAS FINANCIERAS}

CAPDEV 1 DEUDAS FINANCIERAS / EBITDA

CAPDEV 2 DEUDAS FINANCIERAS / EFECTIVO DE LA EXPLOTACIÓN 
Por otra parte, para analizar la rentabilidad y la capacidad de generar riqueza hemos identificado, a su vez, tres subáreas de interés, con 18 ratios, lo que suma un total de 27 indicadores:

d) Rentabilidad económica y financiera.

e) Generación de valor añadido.

f) Productividad del personal.

Para evaluar la rentabilidad económica hemos identificado tres ratios (RENTB 1,3 y 5 ) en los que tres indicadores de resultado se relativizan respecto al activo total. Estos ratios se han descompuesto en sus respectivos elementos de margen (RENTB 2, 4 y 6) y rotación (RENTB 7), que es único para los tres indicadores de rentabilidad definidos. Paralelamente, se han analizado ratios de rentabilidad de los recursos propios (RENTB 8) y el coste de la financiación ajena (RENTB 9), el ratio palanca (RENTB 10) y efecto de apalancamiento financiero (RENTB 11).

\begin{tabular}{|ll|}
\hline \multicolumn{2}{l}{ RENTABILIDAD ECONÓMICA Y FINANCIERA } \\
RENTB 1 & RESULTADO EXPLOTACION / ACTIVO TOTAL \\
RENTB 2 & RESULTADO EXPLOTACION / VENTAS \\
RENTB 3 & (RESULTADO ANTES IMPUESTOS + GASTO FINANCIERO) / ACTIVO TOTAL \\
RENTB 4 & (RESULTADO ANTES IMPUESTOS + GASTO FINANCIERO) / VENTAS \\
RENTB 5 & (RESULTADO DEL EJERCICIO + GASTO FINANCIERO) / ACTIVO TOTAL \\
RENTB 6 & (RESULTADO DEL EJERCICIO + GASTO FINANCIERO) / VENTAS \\
RENTB 7 & VENTAS / ACTIVO TOTAL \\
RENTB 8 & RESULTADO DEL EJERCICIO / PATRINOMIO NETO \\
RENTB 9 & GASTO FINANCIERO/DEUDAS FINANCIERAS \\
RENTB 10 & DEUDAS FINANCIERAS / PATRIMONIO NETO (RATIO PALANCA) \\
RENTB 11 & APALANCAMIENTO FINANCIERO \\
\hline
\end{tabular}

Las singularidades del modelo cooperativo de trabajo asociado, donde el factor trabajo y capital se unen con objetivos compartidos, exigía buscar otros instrumentos alternativos para evaluar la capacidad de generación de riqueza. Con este objetivo se ha determinado el valor añadido bruto generado por las empresas (Valor Añadido Generado $=$ Resultado antes de Impuestos + Gastos de personal + Gasto financiero + Amortizaciones).

Calculado el valor añadido generado, se han construido tres indicadores que relativizan el total de valor añadido generado respecto a activo total, ventas y número de empleados (VAÑAD 1, 2 y 3 respectivamente).

GENERACION DE VALOR AÑADIDO

VAÑAD 1 VALOR AÑADIDO / ACTIVO TOTAL

VAÑAD 2 VALOR AÑADIDO / VENTAS

VAÑAD 3 VALOR AÑADIDO / NÚMERO DE EMPLEADOS 
Finalmente, teniendo presente que el objeto de estudio son las cooperativas industriales de trabajo asociado, en las que la mayoría del factor trabajo comparte la condición de aportante de capital, hemos considerado de interés analizar indicadores que evalúen la productividad del factor trabajo en ambos tipos de organizaciones.

PRODUCTIVIDAD DEL PERSONAL

PRODT 1 GASTO PERSONAL / VALOR AÑADIDO

PRODT 2 GASTO PERSONAL / VENTAS

PRODT 3 VENTAS / NÚMERO DE EMPLEADOS

PRODT 4 RESULTADO EXPLOTACION / NÚMERO DE EMPLEADOS

\section{4.- Resultados del estudio}

El análisis comparado de los datos procedentes de las cuentas anuales de los ejercicios $2010 \mathrm{y}$ 2011 para cada modelo de empresa e indicador elaborado (27 indicadores) supone un total de 54 variables. Inicialmente, en el caso de las sociedades de capital, donde la dispersión de los datos obtenidos resultaba muy superior se propuso eliminar los valores más extremos de los ratios elaborados a fin de no distorsionar los resultados obtenidos.

Sin embargo, dado que los resultados estadísticos no variaron de forma significativa, finalmente se decidió realizar el estudio con la totalidad de las observaciones obtenidas. A fin de comprobar el cumplimiento de la hipótesis de normalidad, sometimos las 54 variables construidas al test de Kolmogorov-Smirnov y Shapiro-Wilk (ver Anexo). Los resultados del test rechazan la hipótesis de normalidad, por lo que posteriormente hemos aplicado técnicas de estadística de contraste no paramétrica.

a) Solvencia a CP (Liquidez)

Los resultados del análisis descriptivo por muestras son los siguientes: 


\section{Tabla 2. Estadísticos descriptivos de solvencia a corto plazo}

\begin{tabular}{|l|l|rr|rr|rr|}
\hline & \multicolumn{2}{|c|}{ SOLVCP 1 } & \multicolumn{2}{c|}{ SOVLCP 2 } & \multicolumn{2}{c|}{ SOLVCP 3 } \\
& & $\mathbf{2 0 1 0}$ & $\mathbf{2 0 1 1}$ & $\mathbf{2 0 1 0}$ & $\mathbf{2 0 1 1}$ & $\mathbf{2 0 1 0}$ & $\mathbf{2 0 1 1}$ \\
\hline SA+SL & N Válidos & 337 & 337 & 337 & 337 & 337 & 337 \\
& Perdidos & 0 & 0 & 0 & 0 & 0 & 0 \\
& Media & 1,7806 & 1,7277 & 1,3045 & 1,2288 & 0,1496 & 0,1440 \\
& Media recortada al 5\% & 1,6426 & 1,5934 & 1,1820 & 1,1142 & 0,1058 & 0,0968 \\
& Mediana & 1,4310 & 1,4291 & 1,0466 & 0,9877 & 0,0504 & 0,0353 \\
& Desviación estándar & 1,2267 & 1,1868 & 1,0123 & 0,9648 & 0,2698 & 0,2914 \\
& Mínimo & 0,1204 & 0,1844 & 0,0685 & 0,0761 & 0,0000 & 0,0000 \\
& Máximo & 10,2639 & 9,5523 & 8,6785 & 8,2609 & 2,4086 & 2,6660 \\
\hline COOP & N Válidos & 50 & 50 & 50 & 50 & 50 & 50 \\
& Perdidos & 0 & 0 & 0 & 0 & 0 & 0 \\
& Media & 2,3429 & 2,0006 & 1,9198 & 1,5584 & 0,2529 & 0,2250 \\
& Media recortada al 5\% & 2,0643 & 1,8856 & 1,6533 & 1,4359 & 0,2064 & 0,1731 \\
& Mediana & 1,7568 & 1,5931 & 1,3120 & 1,1158 & 0,0778 & 0,0619 \\
& Desviación estándar & 1,8675 & 1,0667 & 1,8385 & 1,0059 & 0,3577 & 0,3548 \\
& Mínimo & 0,8019 & 0,7870 & 0,4830 & 0,5673 & 0,0001 & 0,0000 \\
& Máximo & 11,2359 & 6,0394 & 10,9489 & 5,2830 & 1,5867 & 1,7215 \\
\hline
\end{tabular}

El análisis descriptivo de los datos de solvencia a corto plazo muestra medias, medias recortadas y medianas superiores en las cooperativas en cualquiera de los tres ratios construidos y en los dos años observados. Los tres ratios estudiados solvencia a corto, prueba ácida y disponibilidad nos permiten identificar una situación más fortalecida en el ámbito cooperativo en relación a la capacidad de atender las deudas de corto plazo. En el caso del ratio de solvencia a corto plazo (SOLVCP1) el $18 \%$ de las sociedades anónimas y limitadas presentaban ratios inferiores a 1 en el ejercicio 2010, es decir mantienen un fondo de maniobra negativo. En el ejercicio 2011 este porcentaje pasó a representar un $23 \%$ de la muestra. Estos datos contrastan con los de las cooperativas donde sólo una (2\% de la muestra) presentaba fondo de rotación negativo en los dos ejercicios estudiados.

Gráficamente se representan las medianas en las que en los dos primeros indicadores se observa una tendencia a la baja y una reducción de las diferencias en el año 2011. 
Medianas SOLVCP1

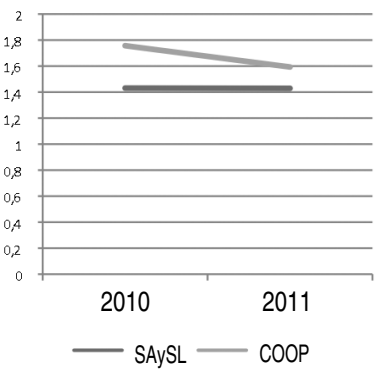

Medianas SOLVCP2

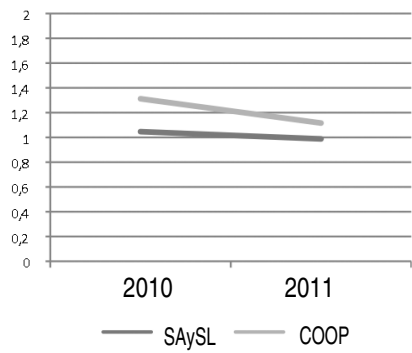

Medianas SOLVCP3

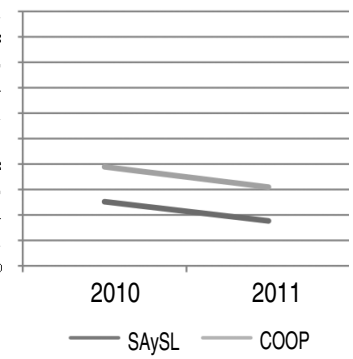

La aplicación del test de Mann-Whitney para muestras independientes rechaza la hipótesis de igualdad de distribución en cada uno de los indicadores y en los dos años observados. Por tanto, las diferencias observadas en los tres ratios de solvencia a corto plazo entre cooperativas y sociedades anónimas y limitadas son estadísticamente significativas.

\section{Tabla 3. Resumen estadístico relativo a ratios de solvencia a corto plazo}

\begin{tabular}{|c|c|c|c|c|c|c|c|}
\hline \multicolumn{2}{|l|}{ Ratio } & $\begin{array}{l}\text { Descriptivo } \\
2010 \\
\text { (Media coop, } \\
\text { respecto de } \\
\text { SAySL) }\end{array}$ & $\begin{array}{l}\text { Descriptivo } \\
2011 \\
\text { (Media coop, } \\
\text { respecto de } \\
\text { SAySL) }\end{array}$ & $\begin{array}{c}\text { Descriptivo } \\
2010 \\
\text { (Mediana } \\
\text { coop.) }\end{array}$ & $\begin{array}{c}\text { Descriptivo } \\
2011 \\
\text { (Mediana } \\
\text { coop.) }\end{array}$ & $\begin{array}{c}\text { Prueba U } \\
\text { de Mann- } \\
\text { Whitney } \\
2010\end{array}$ & $\begin{array}{c}\text { Prueba U } \\
\text { de Mann- } \\
\text { Whitney } \\
2011\end{array}$ \\
\hline SOLVCP 1 & $\mathrm{AC} / \mathrm{PC}$ & Mayor & Mayor & Mayor & Mayor &, $005^{\star \star}$ &, $014^{\star *}$ \\
\hline SOVLCP 2 & (AC-Exist) /PC & Mayor & Mayor & Mayor & Mayor &, $002^{\star \star}$ &, $003^{\star \star}$ \\
\hline SOLVCP 3 & Disp/PC & Mayor & Mayor & Mayor & Mayor &, $019^{* *}$ &, $034^{* *}$ \\
\hline
\end{tabular}

${ }^{* *}$ Sig. $=0,05$

Profundizar en la compleja estructura de las partidas del balance en el corto plazo en la búsqueda de factores que puedan explicar las diferencias identificadas resulta de interés para futuras investigaciones.

b) Estructura Financiera y Solvencia a largo plazo

Con relación a los ratios de solvencia global (SOLVLP1), nuevamente son las cooperativas las empresas que presentan indicadores superiores en términos de media, media recortada y mediana, lo cual denota un mayor nivel de endeudamiento del modelo de sociedades de capital. Es destacable que ninguna de las cooperativas analizadas presenta patrimonio neto negativo, de ahí que el valor 
mínimo de las cooperativas estudiadas supere en todo caso el valor de 1. Esta circunstancia no se cumple en las sociedades anónimas y limitadas en las que el 3,8\% de la muestra (ejercicio 2011) presenta un patrimonio negativo, y donde el valor mínimo ronda en el ambos ejercicios en torno a 0,5, lo que denota que el caso extremo el activo sólo tienen recursos para hacer frente a la mitad de las deudas registradas según valoraciones contables.

\section{Tabla 4. Estadísticos descriptivos de solvencia a largo plazo}

\begin{tabular}{|l|l|rr|rr|rr|rr|}
\hline & \multicolumn{2}{|c|}{ SOLVLP1 } & \multicolumn{2}{c|}{ SOLVLP2 } & \multicolumn{2}{c|}{ SOLVLP3 } & \multicolumn{2}{c|}{ SOLVL4 } \\
& & $\mathbf{2 0 1 0}$ & $\mathbf{2 0 1 1}$ & $\mathbf{2 0 1 0}$ & $\mathbf{2 0 1 1}$ & $\mathbf{2 0 1 0}$ & $\mathbf{2 0 1 1}$ & $\mathbf{2 0 1 0}$ & $\mathbf{2 0 1 1}$ \\
\hline SA+SL & N Válido & 337 & 337 & 337 & 337 & 337 & 337 & 337 & 337 \\
& Perdidos & 0 & 0 & 0 & 0 & 0 & 0 & 0 & 0 \\
& Media & 2,1052 & 2,1050 & 1,2586 & 0,3020 & 0,6872 & 0,6876 & 0,4393 & 0,4449 \\
& Media rec al 5\% & 1,9390 & 1,9350 & 1,0240 & 1,0254 & 0,7527 & 0,7584 & 0,4403 & 0,4468 \\
& Mediana & 1,6729 & 1,6910 & 0,8192 & 0,8120 & 0,7989 & 0,8148 & 0,4667 & 0,4728 \\
& Desviación estándar & 1,2818 & 1,3044 & 5,8479 & 14,3627 & 0,6380 & 0,8226 & 0,2521 & 0,2567 \\
& Mínimo & 0,5244 & 0,4669 & $-69,8690$ & $-184,1900$ & $-8,9980$ & $-12,6980$ & 0,0000 & 0,0000 \\
& Máximo & 10,5990 & 10,5080 & 70,3600 & 66,5410 & 1,0000 & 3,0100 & 0,9409 & 0,9370 \\
\hline COOP & N Válido & 50 & 50 & 50 & 50 & 50 & 50 & 50 & 50 \\
& Perd. & 0 & 0 & 0 & 0 & 0 & 0 & 0 & 0 \\
& Media & 2,3533 & 2,2313 & 0,8557 & 1,0546 & 0,7137 & 0,7262 & 0,3973 & 0,5103 \\
& Media recortada al $5 \%$ & 2,1371 & 2,1128 & 0,9257 & 0,9287 & 0,7288 & 0,7398 & 0,3955 & 0,4021 \\
& Mediana & 1,8304 & 1,8737 & 0,7430 & 0,7988 & 0,7346 & 0,7890 & 0,3562 & 0,4134 \\
& Desviación estándar & 1,5167 & 1,0742 & 1,8157 & 0,8323 & 0,2161 & 0,2057 & 0,2336 & 0,8570 \\
& Mínimo & 1,0990 & 1,1068 & $-9,9562$ & 0,2783 & 0,1263 & 0,2078 & 0,0000 & 0,0000 \\
& Máximo & 2,3533 & 2,2313 & 0,8557 & 1,0546 & 0,7137 & 0,7262 & 0,3973 & 0,5103 \\
\hline
\end{tabular}

Si consideramos los datos de media recortada y mediana del indicador SOLVLP2, en ambos años, por cada euro de patrimonio neto las sociedades anónimas y limitados tienen más volumen de activo a largo plazo, lo cual indica un mayor uso de financiación vía endeudamiento a largo al financiar los activos no corrientes, en este tipo de sociedades. En el ratio de autonomía financiera (SOLVLP3) los resultados no son concluyentes. Si bien la media de los capitales propios respecto a los capitales permanentes es superior en las cooperativas, los valores de la mediana y media recortada son superiores en las sociedades de capital. En consecuencia, dado que el nivel de endeudamiento respecto a los recursos financieros a largo plazo no ofrece diferencias significativas, se podría concluir que el mayor volumen de endeudamiento de las sociedades de capital está relacionado con las diferencias observadas en la estructura de financiación del circulante, pues en el estudio de solvencia a corto plazo se han observado diferencias estadísticamente significativas entre los dos modelos de empresas. 

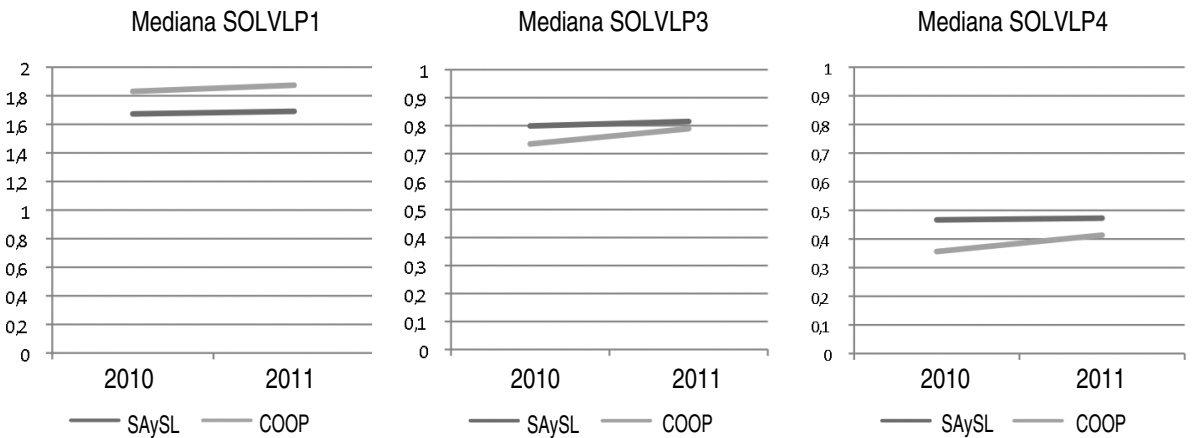

Atendiendo al indicador SOLVLP 4, en el que evaluamos el porcentaje de las deudas financieras sobre el endeudamiento total, de largo y corto plazo, podemos observar que el ratio es menor en términos de mediana y media recortada en las cooperativas. Sin embargo, los datos de media son inversos en los dos años estudiados.

La aplicación del test de Mann-Whitney para muestras independientes no encuentra diferencias significativas al nivel significación del $5 \%$ en ninguna de las variables y años observados, pero sí a un nivel de significación del 10\% para el ratio de solvencia global (SOLVLP1), lo que confirma la fortaleza de las diferencias identificadas a nivel de estadísticos descriptivos en este indicador. Este resultado coincide con los obtenidos en los estudios de Soboh et al. (2011) y Pozuelo et al. (2012). En nuestra opinión, el origen de las diferencias en el nivel de endeudamiento se relaciona con un mayor nivel de endeudamiento en el ciclo corto. Los resultados de nuestro estudio no son concluyentes a la hora de identificar al endeudamiento financiero como factor explicativo del mayor nivel de endeudamiento observado en las sociedades anónimas y limitadas aunque en el análisis descriptivo se han identificado estadísticos de centralidad superiores en las sociedades de corte capitalista. Otra de las causas de las diferencias observadas podría ser un mayor nivel de endeudamiento comercial en anónimas y limitadas.

\section{Tabla 5. Resumen estadístico relativo a ratios de solvencia a largo plazo}

\begin{tabular}{|c|c|c|c|c|c|c|c|c|c|}
\hline \multicolumn{2}{|l|}{ Ratio } & $\begin{array}{c}2010 \\
\text { (Media coop, } \\
\text { respecto de } \\
\text { SAySL) }\end{array}$ & $\begin{array}{c}2011 \\
\text { (Media } \\
\text { coop) }\end{array}$ & $\begin{array}{c}2010 \\
\text { (Media } \\
\text { recortada) }\end{array}$ & $\begin{array}{c}2011 \\
\text { (Media } \\
\text { recortada) }\end{array}$ & $\begin{array}{c}2010 \\
\text { (Mediana } \\
\text { coop.) }\end{array}$ & $\begin{array}{c}2011 \\
\text { (Mediana } \\
\text { coop.) }\end{array}$ & $\begin{array}{c}\text { Prueba U } \\
\text { de Mann- } \\
\text { Whitney } \\
2010\end{array}$ & $\begin{array}{c}\text { Prueba U } \\
\text { de Mann- } \\
\text { Whitney } \\
2011\end{array}$ \\
\hline SOLVLP 1 & $\mathrm{AT} /(\mathrm{PNC}+\mathrm{PC})$ & Mayor & Mayor & Mayor & Mayor & Mayor & Mayor & ,090* & $074^{*}$ \\
\hline SOLVLP 2 & ANC/PN & Menor & Mayor & Menor & Menor & Menor & Menor & ,972 &, 599 \\
\hline SOLVLP 3 & $\mathrm{PN} /(\mathrm{PNC}+\mathrm{PN})$ & Mayor & Mayor & Menor & Menor & Menor & Menor & 228 & ,224 \\
\hline SOLVLP 4 & $\mathrm{DF} /(\mathrm{PNC}+\mathrm{PC})$ & Menor & Mayor & Menor & Menor & Menor & Menor & 219 & 242 \\
\hline
\end{tabular}

${ }^{*}$ Sig. $=0,10$ 


\section{c) Capacidad de devolución de deudas}

En relación a los ratios de capacidad de devolución de deudas financieras, el indicador CAPDEV1 y CAPDEV 2 relacionan las deudas financieras respecto al Ebitda y el efectivo generado por la explotación, respectivamente. Se mide, por tanto, el número de años que serían necesarios para poder devolver la deuda financiera contraída al cierre, al ritmo actual de generación de Ebitda o de efectivo de explotación.

\section{Tabla 6. Estadísticos descriptivos de capacidad de devolución de deudas}

\begin{tabular}{|l|l|rr|rr|}
\hline \multicolumn{2}{|c|}{} & \multicolumn{2}{|c|}{ CAPDEV 1 } & \multicolumn{2}{c|}{ CAPDEV 2 } \\
\hline SA+SL & N Válido & $\mathbf{2 0 1 0}$ & $\mathbf{2 0 1 1}$ & $\mathbf{2 0 1 0}$ & $\mathbf{2 0 1 1}$ \\
& Perdidos & 267 & 265 & 226 & 239 \\
& Media & 70 & 72 & 111 & 98 \\
& Media recortada al 5\% & 8,6708 & 8,3936 & 10,6792 & 16,1740 \\
& Mediana & 3,3686 & 3,2795 & 4,5208 & 4,4268 \\
& Desviación estándar & 3,6224 & 3,1986 & 2,8145 & 2,8588 \\
& Mínimo & 22,1431 & 26,8095 & 35,8842 & 79,4111 \\
& Máximo & 0,0000 & 0,0000 & 0,0000 & 0,0000 \\
\hline COOP & N Válido & 261,4382 & 314,3630 & 471,5636 & 907,2402 \\
& Perdidos & 48 & 45 & 43 & 31 \\
& Media & 2 & 5 & 7 & 19 \\
& Media recortada al 5\% & 3,2098 & 4,7227 & 5,1446 & 6,7672 \\
& Mediana & 2,9706 & 3,0018 & 4,9603 & 5,8929 \\
& Desviación estándar & 2,8778 & 3,1120 & 1,5794 & 2,5288 \\
& Mínimo & 2,5126 & 6,5325 & 10,2080 & 12,5436 \\
& Máximo & 0,0000 & 0,0000 & 0,0000 & 0,0000 \\
& & 11,1756 & 38,7094 & 45,8361 & 62,4211 \\
\hline
\end{tabular}

En el análisis descriptivo se han eliminado todos los casos en las que el Ebitda o el efectivo de explotación resultaban negativos, dado que bajo esas circunstancias la empresa carece en absoluto capacidad de devolución de deudas. Las observaciones consideradas como perdidas por este motivo son más abundantes en el contexto de las sociedades anónimas y limitadas que en las cooperativas. Este hecho es, en sí mismo, revelador de una menor capacidad de devolución de deudas en las sociedades capitalistas. Si atendemos a los resultados de las empresas con Ebitda positivo, los datos del indicador CAPDEV1 son menores en las cooperativas. Esto es, las sociedades cooperativas necesitan menos años que las de capital para la devolución de la deuda financiera contraída al cierre. Los resultados son especialmente diferenciados en términos de media. Las diferencias observadas se reducen de modo claro al trabajar con medias recortadas y medianas, especialmente en el ejercicio 2011. 
Por otra parte, al evaluar la capacidad de devolución en términos de efectivo CAPDEV 2, los resultados son más confusos, dado que las cooperativas ofrecen mejor capacidad de devolución en términos de media y mediana, pero el resultado se altera a favor de las anónimas y limitadas al considerar la media recortada.

La aplicación del test de Mann-Whitney no encuentra diferencias significativas al nivel significación del 10\%. En consecuencia, según las evidencias obtenidas, no podemos constatar diferencias estadísticamente significativas en términos de capacidad de devolución de deudas financieras entre las sociedades cooperativas y las capitalistas industriales.

\section{Tabla 7. Resumen estadístico relativo a ratios sobre capacidad de devolución de deudas}

\begin{tabular}{|c|c|c|c|c|c|c|c|c|c|}
\hline \multicolumn{2}{|l|}{ Ratio } & $\begin{array}{l}2010 \\
\text { (Media coop, } \\
\text { respecto de } \\
\text { SAySL) }\end{array}$ & $\begin{array}{c}2011 \\
\text { (Media } \\
\text { coop) }\end{array}$ & $\begin{array}{c}2010 \\
\text { (Media } \\
\text { recortada) }\end{array}$ & $\begin{array}{c}2011 \\
\text { (Media } \\
\text { recortada) }\end{array}$ & $\begin{array}{c}2010 \\
\text { (Mediana } \\
\text { coop.) }\end{array}$ & $\begin{array}{c}2011 \\
\text { (Mediana } \\
\text { coop.) }\end{array}$ & $\begin{array}{c}\text { Prueba U } \\
\text { de Mann- } \\
\text { Whitney } \\
2010\end{array}$ & $\begin{array}{c}\text { Prueba U } \\
\text { de Mann- } \\
\text { Whitney } \\
2011\end{array}$ \\
\hline CAPDEV 1 & DF/EBITDA & Menor & Menor & Menor & Menor & Menor & Menor & ,442 & ,276 \\
\hline CAPDEV 2 & DF/EfExpl & Menor & Menor & Mayor & Mayor & Menor & Menor & ,352 & 291 \\
\hline
\end{tabular}

${ }^{*}$ Sig. $=0,10$

d) Rentabilidad económica y financiera

En relación a los ratios de rentabilidad económica, en los tres indicadores utilizados que relacionan variables de excedente respecto al activo (RENTB 1, RENTB 3 y RENTB 5) el modelo cooperativo ofrece mejores datos, tanto en términos de media como de mediana. 
Tabla 8. Estadísticos descriptivos de indicadores de rentabilidad económica

\begin{tabular}{|l|l|rr|rr|rr|}
\hline & & \multicolumn{2}{|c|}{ RENTB 1 } & \multicolumn{2}{c|}{ RENTB 3 } & \multicolumn{2}{c|}{ RENTB 5 } \\
& & $\mathbf{2 0 1 0}$ & $\mathbf{2 0 1 1}$ & $\mathbf{2 0 1 0}$ & $\mathbf{2 0 1 1}$ & $\mathbf{2 0 1 0}$ & $\mathbf{2 0 1 1}$ \\
\hline SA+SL & N Válidos & 337 & 337 & 337 & 337 & 337 & 337 \\
& Perdidos & 0 & 0 & 0 & 0 & 0 & 0 \\
& Media & 0,0140 & 0,0069 & 0,0193 & 0,0100 & 0,0152 & 0,0071 \\
& Mediana & 0,0216 & 0,0216 & 0,0252 & 0,0263 & 0,0247 & 0,0257 \\
& Desviación estándar & 0,1670 & 0,1339 & 0,1715 & 0,1351 & 0,1634 & 0,1302 \\
& Mínimo & $-2,3056$ & $-1,4586$ & $-2,3056$ & $-1,4586$ & $-2,3056$ & $-1,4586$ \\
& Máximo & 0,6139 & 0,4660 & 0,8147 & 0,4671 & 0,7851 & 0,4054 \\
\hline COOP & N Válidos & 50 & 50 & 50 & 50 & 50 & 50 \\
& Perdidos & 0 & 0 & 0 & 0 & 0 & 0 \\
& Media & 0,0313 & 0,0345 & 0,0361 & 0,0391 & 0,0357 & 0,0383 \\
& Mediana & 0,0245 & 0,0284 & 0,0283 & 0,0344 & 0,0292 & 0,0335 \\
& Desviación estándar & 0,0400 & 0,0478 & 0,0399 & 0,0500 & 0,0377 & 0,0496 \\
& Mínimo & $-0,0703$ & $-0,0697$ & $-0,0804$ & $-0,0666$ & $-0,0691$ & $-0,0666$ \\
& Máximo & 0,1753 & 0,2606 & 0,1595 & 0,2718 & 0,1553 & 0,2718 \\
\hline
\end{tabular}

Las diferencias son sensiblemente mayores en los datos de media que en los de mediana. Además, estas diferencias son mayores en los tres indicadores en el ejercicio 2011. Las mayores diferencias se observan en el indicador RENTB5. La fiscalidad más favorable en el modelo cooperativo puede ser un factor para que las diferencias aumenten entre cooperativas y sociedades anónimas y limitadas con los resultados después de impuestos.
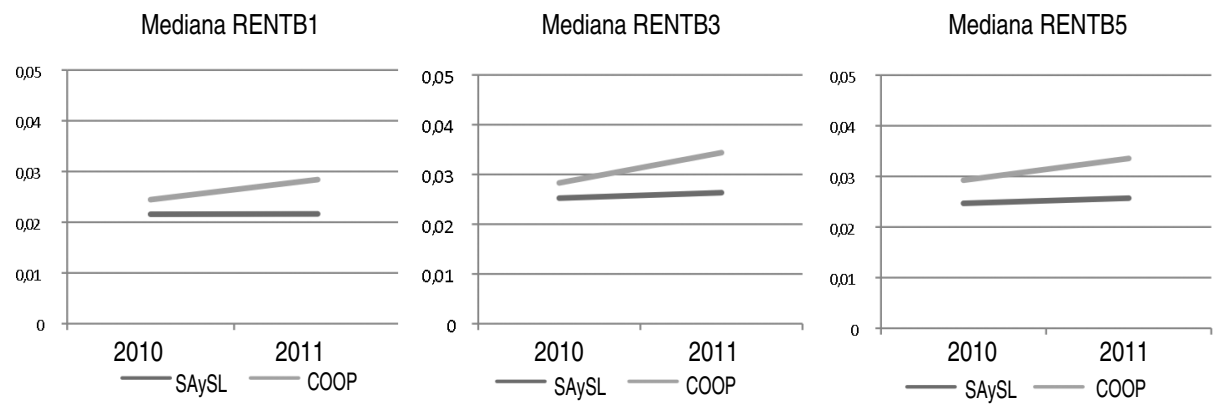
El análisis estadístico de contraste no ofrece resultados significativos a un nivel de significación de $10 \%$. Por tanto, nuestros resultados no identifican diferencias significativas de distribución entre ambas muestras en términos de rentabilidad económica. Los resultados coinciden con los del trabajo de Hind (1994). No obstante, las mayores diferencias observadas en el análisis descriptivo para el ejercicio 2011 se confirman con los datos de las pruebas estadísticas en las que la significación de la aplicación del test de Mann-Whitney produce valores entorno al $11 \%$ en los indicadores RENTB 3 y RENTB 5 en el ejercicio 2011.

\section{Tabla 9. Resumen estadístico relativo a ratios de rentabilidad económica}

\begin{tabular}{|c|c|c|c|c|c|c|c|}
\hline \multicolumn{2}{|l|}{ Ratio } & $\begin{array}{c}\text { Descriptivo } \\
2010 \\
\text { (Media coop, } \\
\text { respecto de } \\
\text { SAySL) }\end{array}$ & $\begin{array}{l}\text { Descriptivo } \\
2011 \\
\text { (Media coop, } \\
\text { respecto de } \\
\text { SAySL) }\end{array}$ & $\begin{array}{l}\text { Descriptivo } \\
2010 \\
\text { (Mediana } \\
\text { coop.) }\end{array}$ & $\begin{array}{c}\text { Descriptivo } \\
2011 \\
\text { (Mediana } \\
\text { coop.) }\end{array}$ & $\begin{array}{c}\text { Prueba U } \\
\text { de Mann- } \\
\text { Whitney } \\
2010\end{array}$ & $\begin{array}{c}\text { Prueba U } \\
\text { de Mann- } \\
\text { Whitney } \\
2011\end{array}$ \\
\hline RENTB 1 & RdoExpl/AT & Mayor & Mayor & Mayor & Mayor & ,360 & 160 \\
\hline RENTB 3 & $(\mathrm{RAl}+\mathrm{GF}) / \mathrm{AT}$ & Mayor & Mayor & Mayor & Mayor & 284 & 113 \\
\hline RENTB 5 & (Rdo+GF)AT & Mayor & Mayor & Mayor & Mayor & 189 & 109 \\
\hline
\end{tabular}

${ }^{\star}$ Sig. $=0,10$

Si estudiamos los descriptivos de los factores de margen y rotación implícitos en los tres indicadores de rentabilidad económica, podemos observar la superioridad de los márgenes de las cooperativas respecto a las sociedades de capital. 


\section{Tabla 10. Estadísticos descriptivos de indicadores de margen y rotación}

\begin{tabular}{|l|l|rr|rr|rr|rr|}
\hline & \multicolumn{2}{|c|}{ RENTB 2 } & \multicolumn{2}{c|}{ RENTB 4 } & \multicolumn{2}{c|}{ RENTB 6 } & \multicolumn{2}{c|}{ RENTB 7 } \\
& & $\mathbf{2 0 1 0}$ & $\mathbf{2 0 1 1}$ & $\mathbf{2 0 1 0}$ & $\mathbf{2 0 1 1}$ & $\mathbf{2 0 1 0}$ & $\mathbf{2 0 1 1}$ & $\mathbf{2 0 1 0}$ & $\mathbf{2 0 1 1}$ \\
\hline SA+SL & N Válido & 337 & 337 & 337 & 337 & 337 & 337 & 337 & 337 \\
& Perdidos & 0 & 0 & 0 & 0 & 0 & 0 & 0 & 0 \\
& Media & 0,0102 & 0,0172 & 0,0167 & 0,0210 & 0,0142 & 0,0201 & 1,0605 & 1,1385 \\
& Mediana & 0,0233 & 0,0224 & 0,0270 & 0,0246 & 0,0253 & 0,0247 & 0,9289 & 0,9911 \\
& Desviación estándar & 0,1646 & 0,4084 & 0,1680 & 0,4092 & 0,1469 & 0,4063 & 0,8195 & 0,7062 \\
& Mínimo & $-1,1690$ & $-1,2604$ & $-1,1691$ & $-1,2531$ & $-1,1690$ & $-1,2531$ & 0,0980 & 0,0473 \\
& Máximo & 1,6258 & 6,9888 & 1,6260 & 6,9888 & 1,1771 & 6,9888 & 11,6890 & 7,0231 \\
\hline COOP & N Válido & 50 & 50 & 50 & 50 & 50 & 50 & 50 & 50 \\
& Perdidos & 0 & 0 & 0 & 0 & 0 & 0 & 0 & 0 \\
& Media & 0,0378 & 0,0439 & 0,0448 & 0,0530 & 0,0446 & 0,0524 & 0,8157 & 1,0141 \\
& Mediana & 0,0266 & 0,0343 & 0,0392 & 0,0391 & 0,0388 & 0,0379 & 0,8007 & 0,8854 \\
& Desviación estándar & 0,0527 & 0,0831 & 0,0535 & 0,0986 & 0,0509 & 0,0988 & 0,3206 & 1,2794 \\
& Mínimo & $-0,0904$ & $-0,2351$ & $-0,1034$ & $-0,2245$ & $-0,0889$ & $-0,2245$ & 0,3139 & 0,1145 \\
& Máximo & 0,2212 & 0,3364 & 0,2012 & 0,4882 & 0,1959 & 0,4927 & 2,0144 & 9,4561 \\
& & & & & & & & & \\
\end{tabular}

Tanto las medias como las medianas de los tres márgenes calculados (RENTB2, RENTB4 y RENTB6) son superiores en las cooperativas en los dos ejercicios observados. En el factor rotación, medido por el indicador RENTB7, se observan resultados opuestos, tanto en términos de media como de mediana. Es decir, las ventas conseguidas por cada euro invertido en el activo son superiores en las sociedades de capital industriales que en las cooperativas industriales.

El análisis estadístico confirma las diferencias observadas en el análisis descriptivo. De este modo, todas las diferencias de distribución de márgenes y rotación resultan significativas en las variables y años observados a un nivel de significación del $5 \%$, con la excepción del margen en términos de resultados antes de impuestos e intereses en 2010 que es significativa al 10\%, y el margen en términos de resultado de explotación en 2010 que no resulta significativo (sig. 0,112). Por lo tanto, según los resultados de nuestro estudio, podemos concluir que existen diferencias significativas entre las empresas industriales cooperativas y capitalistas en la distribución de los márgenes y las rotaciones. Si bien las cooperativas trabajan con márgenes superiores, las ventas por euro invertido en el activo son inferiores a las de las sociedades anónimas y limitadas del País Vasco. En todo caso, las diferencias de actividad identificadas en las dos muestras analizadas y descritas en la tabla 1, suponen una limitación del estudio que podría contribuir a explicar parte de las diferencias observadas. 


\section{Tabla 11. Resumen estadístico relativo a ratios de margen y rotación}

\begin{tabular}{|c|c|c|c|c|c|c|c|}
\hline \multicolumn{2}{|l|}{ Ratio } & \begin{tabular}{|c} 
Descriptivo \\
2010 \\
(Media coop, \\
respecto de \\
SAySL)
\end{tabular} & $\begin{array}{l}\text { Descriptivo } \\
2011 \\
\text { (Media coop, } \\
\text { respecto de } \\
\text { SAySL) }\end{array}$ & $\begin{array}{c}\text { Descriptivo } \\
2010 \\
\text { (Mediana } \\
\text { coop.) }\end{array}$ & $\begin{array}{c}\text { Descriptivo } \\
2011 \\
\text { (Mediana } \\
\text { coop.) }\end{array}$ & $\begin{array}{c}\text { Prueba U } \\
\text { de Mann- } \\
\text { Whitney } \\
2010\end{array}$ & $\begin{array}{c}\text { Prueba U } \\
\text { de Mann- } \\
\text { Whitney } \\
2011\end{array}$ \\
\hline RENTB 2 & RdoExpl/Vtas & Mayor & Mayor & Mayor & Mayor & ,112 &, $015^{\star \star}$ \\
\hline RENTB 4 & (RAl+GF)/Ntas & Mayor & Mayor & Mayor & Mayor &, $064^{*}$ &, $011^{* *}$ \\
\hline RENTB 6 & (Rdo+GF)Vtas & Mayor & Mayor & Mayor & Mayor &, $021^{\star *}$ &, $009^{\star \star}$ \\
\hline RENTB 7 & Vtas/AT & Menor & Menor & Menor & Menor &, $007^{\star *}$ &, $003^{\star *}$ \\
\hline
\end{tabular}

${ }^{*}$ Sig. $=0,10{ }^{*}$ Sig. $=0,05$

Nuevamente, los resultados del análisis descriptivo de los ratios de rentabilidad financiera muestran mejores resultados en las cooperativas que en las sociedades anónimas y limitadas.

\section{Tabla 12. Estadísticos descriptivos de los indicadores de rentabilidad financiera}

\begin{tabular}{|l|l|rr|rr|rr|rr|}
\hline & \multicolumn{2}{|c|}{ RENTB 8 } & \multicolumn{2}{c|}{ RENTB 9 } & \multicolumn{2}{c|}{ RENTB 10 } & \multicolumn{2}{c|}{ RENTB 11 } \\
& & 2010 & $\mathbf{2 0 1 1}$ & $\mathbf{2 0 1 0}$ & $\mathbf{2 0 1 1}$ & 2010 & $\mathbf{2 0 1 1}$ & $\mathbf{2 0 1 0}$ & $\mathbf{2 0 1 1}$ \\
\hline SA+SL & N Válido & 337 & 337 & 337 & 337 & 337 & 337 & 337 & 337 \\
& Perdidos & 0 & 0 & 0 & 0 & 0 & 0 & 0 & 0 \\
& Media & $-0,0519$ & $-0,4071$ & 0,0965 & 0,0850 & 1,3318 & $-0,6118$ & $-0,1035$ & $-0,0044$ \\
& Media rec. 5\% & 0,0407 & 0,0250 & 0,0386 & 0,0503 & 0,9118 & 0,8437 & $-0,0081$ & $-0,0169$ \\
& Mediana & 0,0389 & 0,0411 & 0,0313 & 0,0390 & 0,5485 & 0,4833 & $-0,0008$ & $-0,0007$ \\
& Desv. estándar & 1,3409 & 10,2094 & 0,4329 & 0,2261 & 8,4600 & 28,0484 & 1,2935 & 6,7678 \\
& Mínimo & $-19,8629$ & $-139,6336$ & 0,0000 & 0,0000 & $-98,1622$ & $-440,9870$ & $-19,5394$ & $-46,5867$ \\
& Máximo & 2,7220 & 103,4087 & 5,0218 & 2,7973 & 99,9977 & 94,9526 & 2,7376 & 103,5832 \\
\hline COOPP & N Válido & 50 & 50 & 50 & 50 & 50 & 50 & 50 & 50 \\
& Perdidos & 0 & 0 & 0 & 0 & 0 & 0 & 0 & 0 \\
& Media & 0,0596 & 0,0535 & 0,0748 & 0,1278 & 0,8728 & 0,8609 & 0,0051 & $-0,0055$ \\
& Media rec. 5\% & 0,0565 & 0,0479 & 0,0563 & 0.0625 & 0,5962 & 0,6500 & 0,0006 & $-0,0039$ \\
& Mediana & 0,0578 & 0,0513 & 0,0477 & 0,0469 & 0,4292 & 0,3876 & $-0,0010$ & $-0,0034$ \\
& Desv. estándar & 0,0895 & 0,0924 & 0,1188 & 0,3324 & 1,5493 & 1,3670 & 0,0561 & 0,0359 \\
& Mínimo & $-0,1563$ & $-0,1266$ & 0,0000 & 0,0000 & 0,0000 & 0,0000 & $-0,1108$ & $-0,1396$ \\
& Máximo & 0,4088 & 0,4334 & 0,8295 & 1,7662 & 7,3438 & 7,1538 & 0,3308 & 0,0999 \\
\hline
\end{tabular}


La mediana del indicador RENTB 8, que mide la relación entre el resultado final del ejercicio y patrimonio neto al cierre (ROE- Return on Equity), es 1,89 puntos superior en el ejercicio 2010 y 1,02 puntos superior en el 2011.

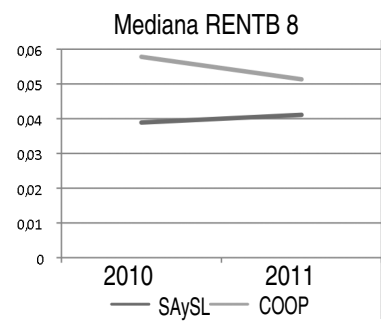

Además, según los datos de la muestra, en el ejercicio 2010 obtuvo pérdidas el $32 \%$ de las sociedades anónimas y limitadas industriales del País Vasco, y el 33\% en 2011. En el caso de las cooperativas, en el ejercicio 2010 sólo tenía pérdidas el 10\%, mientras que en 2011 el porcentaje aumentó hasta el alcanzar 22\%, lejos aún de los datos de las sociedades de capital. La amplia dispersión de los datos en las sociedades de capital deja el valor de la media en negativo en ambos ejercicios. $\mathrm{Si}$ atendemos a la media recortada al $5 \%$, los datos vuelven a ser favorables para las cooperativas, con diferencias de 1,6 y 2,2 puntos en 2010 y 2011, respectivamente. Pero realizado el análisis estadístico, la aplicación del test de Mann-Whitney no considera significativas las diferencias observadas, y por tanto, nuestro trabajo no identifica diferencias de distribución de rentabilidad financiera, estadísticamente significativas, entre cooperativas, y anónimas y limitadas industriales (ver tabla 13).

A través del indicador RENTB 9 estudiamos el coste efectivo de la deuda financiera. Los resultados del estudio señalan un mayor coste de la financiación ajena para las empresas cooperativas, tanto en términos de media recortada al $5 \%$ como en mediana, y en ambos ejercicios. Las diferencias son superiores en el 2010 respecto del 2011. Aplicado el test de Mann-Whitney, las diferencias observadas se consideran significativas. El nivel de significación es superior en el ejercicio 2010 (ver tabla 13). Por tanto, a pesar de los inferiores ratios de endeudamiento de las cooperativas, éstas han de soportar unos costes superiores en la financiación ajena. En nuestra opinión, el uso de la financiación a través de empresas de grupo, más propia de las empresas de corte capitalista, podría ser uno de los factores que justifican parte la diferencia observada. No obstante, se trata de un resultado sorprendente que estimula estudios futuros.

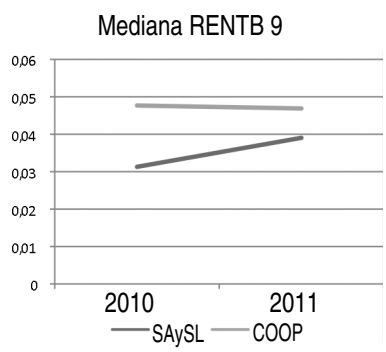


Por su parte, los indicadores RENTB10 y RENTB11 están orientados a definir el posicionamiento de las empresas en cuanto a apalancamiento financiero. El ratio palanca RENTB10 es superior en las capitalistas que en las cooperativas. En consecuencia, se puede decir que el nivel de deudas financieras respecto de cada euro de patrimonio neto es superior en términos de media recortada y mediana en las sociedades anónimas y limitadas industriales. En cambio, el apalancamiento financiero RENTB11 ofrece en ambos modelos valores muy próximos a 0 , tanto en términos de media recortada como de mediana en los dos años observados.

Así, las cooperativas, a pesar de tener mejores indicadores de rentabilidad económica, soportan un coste superior en el endeudamiento, lo que unido a un menor nivel de endeudamiento, neutraliza las posibles diferencias en términos de apalancamiento financiero. Sin embargo, las pruebas de contraste estadístico no paramétrico para muestras independientes, test de Mann-Whitney, no resultan significativas. Por tanto, no encontramos diferencias estadísticamente significativas en los ratios palanca y apalancamiento financiero entre cooperativas, y anónimas y limitadas industriales.

\section{Tabla 13. Resumen estadístico relativo a ratios de rentabilidad financiera}

\begin{tabular}{|c|c|c|c|c|c|c|c|c|c|}
\hline \multicolumn{2}{|l|}{ Ratio } & $\begin{array}{c}\text { Media } \\
2010 \text { (coop, } \\
\text { respecto } \\
\text { de SAySL) }\end{array}$ & $\begin{array}{c}\text { Media } \\
2011 \text { (coop, } \\
\text { respecto } \\
\text { de SAySL) }\end{array}$ & $\begin{array}{c}\text { Media } \\
\text { recortada } \\
2010\end{array}$ & $\begin{array}{c}\text { Media } \\
\text { recortada } \\
2011\end{array}$ & $\begin{array}{c}\text { Mediana } \\
2010\end{array}$ & $\begin{array}{c}\text { Mediana } \\
2011\end{array}$ & $\begin{array}{c}\text { U de } \\
\text { Mann- } \\
\text { Whitney } \\
2010\end{array}$ & $\begin{array}{c}\text { U de } \\
\text { Mann- } \\
\text { Whitney } \\
2011\end{array}$ \\
\hline RENTB 8 & Rdo/PN & Mayor & Mayor & Mayor & Mayor & Mayor & Mayor & ,424 &, 545 \\
\hline RENTB 9 & GF/DF & Menor & Mayor & Mayor & Mayor & Mayor & Mayor &, $001^{\star *}$ &, $051^{*}$ \\
\hline RENTB 10 & DF/PN & Menor & Mayor & Menor & Menor & Menor & Menor & 201 & 493 \\
\hline RENTB 11 & Apalanc $\mathrm{F}^{\circ}$ & Mayor & Menor & Mayor & Mayor & Menor & Menor & ,734 & ,962 \\
\hline
\end{tabular}

${ }^{\star}$ Sig. $=0,10{ }^{*}$ Sig. $=0,05$

\section{e) Generación de valor añadido}

Según los resultados obtenidos en nuestro estudio, las cooperativas industriales del País Vasco ofrecen menor capacidad de generar valor añadido por cada euro invertido en el activo (VAÑAD1). Los tres indicadores de centralidad calculados (media, media recortada y mediana) son mayores en el caso de las sociedades de capital. 
Tabla 14. Estadísticos descriptivos de valor añadido

\begin{tabular}{|l|l|rr|rr|rr|}
\hline & \multicolumn{2}{|c|}{ VAÑAD 1 } & \multicolumn{2}{c|}{ VAÑAD 2 } & \multicolumn{2}{c|}{ VAÑAD 3 } \\
& & 2010 & $\mathbf{2 0 1 1}$ & $\mathbf{2 0 1 0}$ & $\mathbf{2 0 1 1}$ & $\mathbf{2 0 1 0}$ & $\mathbf{2 0 1 1}$ \\
\hline SA+SL & N Válido & 337 & 337 & 337 & 337 & 337 & 337 \\
& Perdidos & 0 & 0 & 0 & 0 & 0 & 0 \\
& Media & 0,3460 & 0,3523 & 0,3569 & 0,3762 & 61,0703 & 60,3022 \\
& Media recortada al 5\% & 0,3134 & 0,3179 & 0,3385 & 0,3170 & 56,6333 & 57,2982 \\
& Mediana & 0,3031 & 0,3004 & 0,3331 & 0,3122 & 54,3889 & 54,7024 \\
& Desviación estándar & 0,2876 & 0,2934 & 0,2249 & 0,9177 & 44,4083 & 33,8982 \\
& Mínimo & $-0,1463$ & $-0,0407$ & $-0,0876$ & $-0,2534$ & $-67,6679$ & $-3,1226$ \\
& Máximo & 2,7407 & 3,0126 & 2,7007 & 16,8789 & 483,2000 & 301,2923 \\
\hline COOPP & N Válido & 50 & 50 & 50 & 50 & 50 & 50 \\
& Perdidos & 0 & 0 & 0 & 0 & 0 & 0 \\
& Media & 0,2797 & 0,2911 & 0,3717 & 0,4099 & 61,3965 & 62,1706 \\
& Media recortada al $5 \%$ & 0,2707 & 0,2801 & 0,3570 & 0,3631 & 59,3175 & 60,0377 \\
& Mediana & 0,2626 & 0,2689 & 0,3383 & 0,3381 & 56,7953 & 59,0694 \\
& Desviación estándar & 0,1206 & 0,1402 & 0,1693 & 0,3627 & 29,6185 & 30,4089 \\
& Mínimo & 0,0667 & 0,0601 & 0,0713 & 0,0223 & 0,0021 & 0,0029 \\
& Máximo & 0,6283 & 0,7613 & 1,0096 & 2,5542 & 225,1652 & 221,3220 \\
\hline
\end{tabular}

En los otros dos ratios, en los que el valor añadido se divide entre el volumen de ventas y el número medio de empleados (VAÑAD2 y 3), los resultados son opuestos al primero de los indicadores. De este modo, son las cooperativas industriales las que generan un mayor valor añadido por cada euro de ventas y por cada empleado, resultado que se ve explicado por los tres indicadores de centralidad calculados. Sin embargo, al aplicar el test de Mann-Whitney las diferencias no resultan significativas, con la excepción del ratio VAÑAD 1 con un nivel de error del 5\%, en uno de los ejercicios (2010).

\section{Tabla 15. Resumen estadístico relativo a ratios de valor añadido}

\begin{tabular}{|c|c|c|c|c|c|c|c|c|c|}
\hline \multicolumn{2}{|l|}{ Ratio } & \multirow{2}{*}{$\begin{array}{c}\text { Media } \\
2010 \text { (coop, } \\
\text { respecto } \\
\text { de SAySL) } \\
\text { Menor }\end{array}$} & $\begin{array}{c}\text { Media } \\
2011 \text { (coop, } \\
\text { respecto } \\
\text { de SAySL) }\end{array}$ & $\begin{array}{c}\text { Media } \\
\text { recortada } \\
2010\end{array}$ & $\begin{array}{c}\text { Media } \\
\text { recortada } \\
2011\end{array}$ & $\begin{array}{c}\text { Mediana } \\
2010\end{array}$ & $\begin{array}{c}\text { Mediana } \\
2011\end{array}$ & \multirow{2}{*}{$\begin{array}{c}\text { U de } \\
\text { Mann- } \\
\text { Whitney } \\
2010 \\
009^{\star *}\end{array}$} & \multirow{2}{*}{$\begin{array}{r}\text { U de } \\
\text { Mann- } \\
\text { Whitne } \\
2011 \\
, 202\end{array}$} \\
\hline VAÑAD 1 & VA/AT & & Menor & Menor & Menor & Menor & Menor & & \\
\hline VAÑAD 2 & VA/Vtas & Mayor & Mayor & Mayor & Mayor & Mayor & Mayor &, 510 &, 143 \\
\hline VAÑAD 3 & VA/NEmpl & Mayor & Mayor & Mayor & Mayor & Mayor & Mayor & 210 & 265 \\
\hline
\end{tabular}

${ }^{*}$ Sig. $=0,05$ 


\section{f) Productividad}

El objetivo de este último bloque de análisis de resultados consiste en evaluar las diferencias en la productividad de los empleados. La singularidad de la posición de la mayoría de los empleados de las cooperativas industriales de trabajo asociado, en su doble condición de socio y aportante de capital, justifica el estudio de los cuatro ratios empleados. En los dos primeros indicadores se ha calculado la proporción que los gastos de personal representan sobre las ventas y sobre el valor añadido generado. En el primer caso (PRODT1), los tres estadísticos de centralidad elaborados son menores en las cooperativas industriales, lo que indica una mayor capacidad de generar valor por cada euro invertido en personal en las cooperativas.

\section{Tabla 16. Estadísticos descriptivos de productividad}

\begin{tabular}{|l|l|rr|rr|rr|rr|}
\hline & \multicolumn{2}{|c|}{ PRODT 1 } & \multicolumn{2}{c|}{ PRODT 2 } & \multicolumn{2}{c|}{ PRODT 3 } & \multicolumn{2}{c|}{ PRODT 4 } \\
& & $\mathbf{2 0 1 0}$ & $\mathbf{2 0 1 1}$ & $\mathbf{2 0 1 0}$ & $\mathbf{2 0 1 1}$ & $\mathbf{2 0 1 0}$ & $\mathbf{2 0 1 1}$ & $\mathbf{2 0 1 0}$ & $\mathbf{2 0 1 1}$ \\
\hline SA+SL & N Válido & 337 & 337 & 337 & 337 & 337 & 337 & 337 & 337 \\
& Perdidos & 0 & 0 & 0 & 0 & 0 & 0 & 0 & \\
& Media & 1,1038 & 0,9940 & 0,2906 & 0,3104 & 230,12 & 247,90 & 7,30 & 5,69 \\
& Media recort al 5\% & 0,7991 & 0,8267 & 0,2752 & 0,2662 & 194,96 & 208,15 & 5,15 & 4,52 \\
& Mediana & 0,7744 & 0,7914 & 0,2658 & 0,2526 & 164,87 & 174,09 & 3,65 & 3,51 \\
& Desviación estándar & 5,3011 & 1,4821 & 0,1716 & 0,5511 & 246,93 & 277,03 & 30,01 & 25,17 \\
& Mínimo & $-14,7625$ & $-3,7398$ & 0,0273 & 0,0222 & 9,51 & 3,60 & $-94,76$ & $-138,41$ \\
& Máximo & 95,9109 & 22,1271 & 1,3634 & 9,8901 & 2689,51 & 2482,21 & 225,06 & 197,23 \\
\hline COOP & N Válido & 50 & 50 & 50 & 50 & 50 & 50 & 50 & 50 \\
& Perdidos & 0 & 0 & 0 & 0 & 0 & 0 & 0 & 0 \\
& Media & 0,7144 & 0,7487 & 0,2709 & 0,3027 & 211,23 & 292,06 & 8,96 & 8,01 \\
& Media recort al 5\% & 0,7108 & 0,7307 & 0,2531 & 0,2653 & 185,86 & 195,07 & 7,39 & 7,71 \\
& Mediana & 0,7114 & 0,7155 & 0,2420 & 0,2513 & 168,96 & 176,31 & 4,78 & 5,73 \\
& Desviación estándar & 0,1368 & 0,2055 & 0,1565 & 0,2655 & 178,89 & 597,18 & 13,52 & 11,79 \\
& Mínimo & 0,4425 & 0,4610 & 0,0406 & 0,0117 & 0,0039 & 0,0029 & $-9,95$ & $-30,35$ \\
& Máximo & 1,1288 & 1,8133 & 0,9566 & 1,8176 & 1069,42 & 4213,16 & 73,00 & 48,91 \\
\hline
\end{tabular}

Al relativizar sobre ventas (PRODT2), se obtienen resultados en la misma dirección pero con menores diferencias entre las cooperativas y las sociedades anónimas y limitadas, especialmente en el ejercicio 2011 donde los resultados son muy similares en cuanto a mediana y media recortada. 
Mediana PRODT1

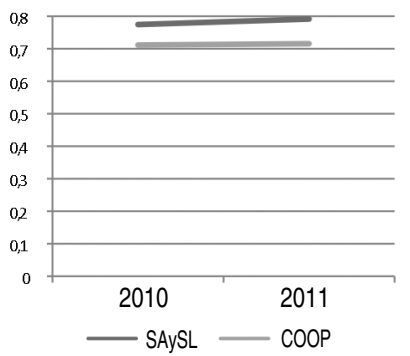

Mediana PRODT2

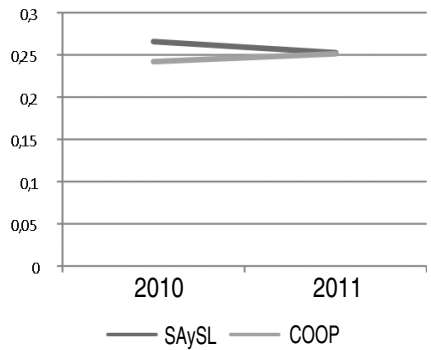

Así, las diferencias observadas solamente son estadísticamente significativas a un nivel de significación del $5 \%$ en el ratio PRODT1 en ambos ejercicios (ver tabla 17).

Por otro lado, los resultados de ventas por empleado, evaluadas en el indicador PRODT 3, tampoco son concluyentes. Las sociedades de capital tienen una media recortada superior, mientras que la media y la mediana es superior en las cooperativas industriales. Como era de esperar, las pruebas de contraste estadístico no resultan significativas (ver tabla 17).

Finalmente, al evaluar el resultado de explotación generado por cada empleado (PRODT4), las cooperativas industriales obtienen resultados sensiblemente superiores en los tres valores de media, media recortada y mediana, y las diferencias observadas son estadísticamente significativas al $10 \%$.

\section{Tabla 17. Resumen estadístico relativo a ratios de productividad}

\begin{tabular}{|c|c|c|c|c|c|c|c|c|c|}
\hline \multicolumn{2}{|l|}{ Ratio } & $\begin{array}{c}\text { Media } \\
2010 \text { (coop, } \\
\text { respecto } \\
\text { de SAySL) }\end{array}$ & $\begin{array}{c}\text { Media } \\
2011 \text { (coop, } \\
\text { respecto } \\
\text { de SAySL) }\end{array}$ & $\begin{array}{c}\text { Media } \\
\text { recortada } \\
2010\end{array}$ & $\begin{array}{l}\text { Media } \\
\text { recortada } \\
2011\end{array}$ & $\begin{array}{c}\text { Mediana } \\
2010\end{array}$ & $\begin{array}{c}\text { Mediana } \\
2011\end{array}$ & $\begin{array}{c}\text { U de } \\
\text { Mann- } \\
\text { Whitney } \\
2010\end{array}$ & $\begin{array}{c}\text { U de } \\
\text { Mann- } \\
\text { Whitney } \\
2011\end{array}$ \\
\hline PRODT 1 & GP/VA & Menor & Menor & Menor & Menor & Menor & Menor &, $012^{* \star}$ &, $013^{\star *}$ \\
\hline PRODT 2 & GP/Vtas & Menor & Menor & Menor & Menor & Menor & Menor & 288 & ,954 \\
\hline PRODT 3 & Vtas/Nempl & Menor & Mayor & Menor & Mayor & Mayor & Mayor & ,646 & ,812 \\
\hline PRODT 4 & RdoExpl/NEmpl & Mayor & Mayor & Mayor & Mayor & Mayor & Mayor & ,090* &, $061^{*}$ \\
\hline
\end{tabular}

${ }^{*}$ Sig. $=0,10$ * Sig. $=0,05$

A modo de resumen, mostramos los ratios en los que hemos identificado diferencias de distribución estadísticamente significativas en alguno de los ejercicios (tabla 18). 


\section{Tabla 18. Listado de ratios con contraste significativo en alguno de los ejercicios}

\begin{tabular}{|c|c|c|c|}
\hline \multicolumn{2}{|c|}{ Ratio } & \multirow{2}{*}{$\begin{array}{c}\text { Prueba U de } \\
\text { Mann-Whitney } 2010 \\
, 005^{\star \star}\end{array}$} & \multirow{2}{*}{$\begin{array}{c}\text { Prueba U de } \\
\text { Mann-Whitney } 2011 \\
, 014^{* *}\end{array}$} \\
\hline SOLVCP 1 & $A C / P C$ & & \\
\hline SOVLCP 2 & (AC-Exist)/PC &, $002^{\star *}$ &, $003^{\star *}$ \\
\hline SOLVCP 3 & Disp/PC &, $019^{\star *}$ &, $034^{\star *}$ \\
\hline SOLVLP 1 & $\mathrm{AT} /(\mathrm{PNC}+\mathrm{PC})$ &, $090^{*}$ &, $074^{*}$ \\
\hline RENTB 2 & RdoExpl/Vtas & ,112 &, $015^{\star *}$ \\
\hline RENTB 4 & $(\mathrm{RAl}+\mathrm{GF}) / \mathrm{Vtas}$ &, $064^{*}$ &, $011^{\star \star}$ \\
\hline RENTB 6 & (Rdo+GF)Vtas &, $021^{\star *}$ &, $009^{\star *}$ \\
\hline RENTB 7 & Vtas/AT &, $007^{\star *}$ &, $003^{\star *}$ \\
\hline RENTB 9 & GF/DF &, $001^{* *}$ &, $051^{*}$ \\
\hline VAÑAD 1 & VA/AT &, $009^{*}$ & ,202 \\
\hline PRODT 1 & GP/VA &, $012^{\star *}$ &, $013^{\star *}$ \\
\hline PRODT 4 & RdoExpl/NEmpl &, $090^{\star}$ &, $061^{*}$ \\
\hline
\end{tabular}

${ }^{*}$ Sig. $=0,10$ ** Sig. $=0,05$

Por tanto, observamos que las cooperativas analizadas presentan valores mejores que las sociedades capitalistas en muchos indicadores que en algunos casos resultan, además, diferencias significativas, tanto en términos de solvencia, como de rentabilidad, valor añadido y productividad por empleado. 


\section{5.- Reflexiones finales}

Las sociedades cooperativas son agentes centrales de la economía social que contribuyen de modo muy significativo a la generación de empleo y riqueza, tanto en España como en Europa, y muy especialmente en Euskadi. La literatura previa ha asociado las singularidades de las cooperativas en cuanto a la configuración de sus capitales propios como una limitación de la capacidad para acceder a mayor financiación, limitación que las diferentes legislaciones tratan de contrarrestar imponiendo mayores exigencias de retención de excedentes respecto de las sociedades de capital. En el caso de las cooperativas de trabajo asociado otro elemento diferenciador tiene que ver con el control de la toma de decisiones por parte de los propios trabajadores, que junto a la asunción de los principios cooperativos, contribuye a justificar un mejor comportamiento en los indicadores de mantenimiento de empleo en los periodos de crisis.

En este sentido, el trabajo presenta un estudio de las diferencias en cuanto estructura financiera, solvencia, rentabilidad y capacidad de generar riqueza respecto a las sociedades de capital. Para ello se analizan las cooperativas de trabajo asociado de más de 50 trabajadores del sector industrial en comparación con sociedades de capital de similar tamaño y actividad de la Comunidad Autónoma del País Vasco, sobre las que no hemos encontrado estudios anteriores. Además, se han utilizado tanto indicadores económico-financieros tradicionales como otros más adecuados a la realidad cooperativa, como es el cálculo del valor añadido.

Los resultados obtenidos muestran que las cooperativas de trabajo estudiadas ofrecen indicadores similares o superiores a las sociedades anónimas y limitadas. Las cooperativas presentan un menor nivel de endeudamiento y, por tanto, un mayor nivel de solvencia global, resultados que están en línea con los obtenidos por Chaddad (2001), Soboh et al. (2012) y Pozuelo et al. (2012). Ello sugiere que las políticas de fortalecimiento de la autofinanciación impuesta legalmente consiguen contrarrestar la tradicional limitación en la captación de capital propio, dando como resultado unas estructuras financieras globales más fortalecidas que las sociedades de capital.

Además, hemos avanzado identificando el origen de los diferentes niveles de endeudamiento observados. Así, hemos podido constatar que las diferencias se relacionan con un menor nivel de endeudamiento en el ciclo corto, de modo que la causa de las diferencias observadas podría ser un mayor nivel de endeudamiento comercial en sociedades anónimas y limitadas. En todo caso, los resultados no son concluyentes a la hora de identificar al endeudamiento financiero como factor explicativo del menor nivel de endeudamiento global observado en las cooperativas. 
Por otra parte, la consecución de adecuadas tasas de rentabilidad es otro de los pilares básicos para la supervivencia de cualquier negocio. Los resultados del análisis, aunque no resultan estadísticamente significativos, sugieren tasas de rentabilidad económica y financiera superiores en las cooperativas, lo que es contradictorio con la mayoría de los trabajos precedentes, aunque coinciden con los observados por Hind (1994).

Se han observado diferencias significativas en indicadores de productividad, que muestran una mayor capacidad de generar valor por cada euro invertido en personal en las cooperativas. A su vez, el resultado de explotación generado por cada empleado de las cooperativas es superior al de las sociedades de capital.

Por lo tanto, a pesar de que las cooperativas se asocian a mejores niveles de mantenimiento de empleo en épocas de crisis, esta política no parece tener incidencia en las tasas de rentabilidad observadas en nuestro estudio. Esta situación podría explicarse a través de los ajustes en niveles salariales, tradicionales en las cooperativas de trabajo asociado, sin incidencia en despidos de socios.

Concluimos que, a pesar de la convulsión que ha supuesto la caída de Fagor Electrodomésticos, una de las empresas referentes de cooperativismo vasco, éste presenta unos indicadores positivos que le permitirán continuar siendo una fuente fundamental de generación de riqueza y empleo en la CAPV.

En todo caso, el trabajo analiza únicamente datos de dos ejercicios, de modo que no se pueden extraer mayores conclusiones sobre la evolución en el tiempo de los indicadores observados. Futuros trabajos podrán estudiar los efectos que la crisis global ha ocasionado entre ambos colectivos con series temporales más amplias. También resultará de interés ahondar en el análisis individualizado de las características y políticas singulares de las cooperativas como causa de las diferencias observadas.

\section{6.- Bibliografía}

AMAT, O. \& PERRAMON, J. (2011): "High-growth cooperatives: financial profile and key factors for competitiveness", CIRIEC-España, Revista de Economía Pública, Social y Cooperativa, 73, 8198.

ANDICOECHEA ARONDO, L. \& ZUBIAURRE ARTOLA, M. (2012): "Equity-liability accounting debate in worker co-operative entities members' shares", Journal of Co-operative Accounting and Reporting, 1(1), 28-46. 
ARIAS, B. \& MONTEGUT, Y. (2012): "Los efectos de la nueva normativa contable en las cooperativas", Revista De Contabilidad y Dirección, 14, 183-201.

ASOCIACIÓN ESPAÑOLA DE CONTABILIDAD Y ADMINISTRACIÓN DE EMPRESAS, AECA (2009): Documento 1, Fondos propios en las cooperativas, Comisión Contabilidad de Cooperativas, Madrid, AECA.

ASOCIACIÓN ESPAÑOLA DE CONTABILIDAD Y ADMINISTRACIÓN DE EMPRESAS, AECA (2010): Documento 2, El fondo de educación, formación y promoción, Comisión Contabilidad de cooperativas, Madrid.

BARTLETT, W.J., CABLE, J., ESTRIN, S., JONES, D.C. \& SMITH, S.C. (1992): "Labor-managed cooperatives and private firms in North Central Italy: an empirical comparison", Industrial and Labor Relations Review, 46, 103-118.

BAKAIKOA, B., ERRASTI, A. \& BEGIRISTAIN, A. (2004): "Governance of the Mondragon Corporación Cooperativa", Annals of Public and Cooperative Economics, 75(1), 61-87.

BASTIDA VIALCANET, R. \& CARRERAS ROIG, L. (2013): "Empirical study about the effects of the application of IAS 32 in co-operatives", Intangible Capital, 9(2), 392-419.

BEL DURÁN, P. \& FERNÁNDEZ GUADAÑO, J. (2002): "La financiación propia y ajena de las sociedades cooperativas", CIRIEC-España, Revista de Economía Pública, Social y Cooperativa, 42, 101-130.

BEN-NER, A. (1988): "Comparative empirical observations on worker-owned and capitalist firms", International Journal of Industrial Organization, 6(1), 7-31.

BIRCHALL, J. \& KETILSON, L.H. (2009): Resilience of the cooperative business model in times of crisis, International Labour Organisation.

BONIN, J.P., JONES, D.C. \& PUTTERMAN, L. (1993): "Theoretical and empirical studies of producer cooperatives: Will ever the twain meet?", Journal of Economic Literature, 31, 1290-1290.

CABALEIRO, M.J., RUIZ BLANCO, S. \& FERNÁNDEZ-FEIJÓO, B. (2010): "Las aportaciones obligatorias al capital social en la reforma contable cooperativa", CIRIEC-España, Revista de Economía Pública, Social y Cooperativa, 69, 217-244.

CALDERÓN MILÁN, B. \& CALDERÓN MILÁN, M.J. (2012): "Cómo afrontan la crisis las cooperativas en España: Comparativa de trayectorias laborales a partir de la muestra continua de vidas laborales", CIRIEC-España, Revista de Economía Pública, Social y Cooperativa, 76, 5-26.

CANTARERO, S., GONZÁLEZ-LOUREIRO, M. \& PUIG, F. (2013): "El efecto 'economía social' en la supervivencia empresarial", CIRIEC-España. Revista de Economía Pública, Social y Cooperativa, 78, 175-202.

CUBEDO TORTONDA, M. (2007): "El régimen económico de las sociedades cooperativas: Situación actual y apuntes para una reforma", CIRIEC-España, Revista de Economía Pública, Social y Cooperativa, 58, 161-187. 
CHADDAD, F.R. (2001): Financial constraints in United States agricultural cooperatives: Theory and panel data econometric evidence. Ph.D., University of Missouri - Columbia. ProQuest Dissertations and Theses.

COMISIÓN EUROPEA (2010): Europa 2020, Disponible en http://eur lex.europa.eu/LexUriServ/LexUriServ.do?uri=COM:2010:2020:FIN:ES:PDF

COOPERATIVES EUROPE (2010): Co-operatives will contribute to the success of the UE 2020 strategy, Cooperatives Europe Communications.

DÍAZ-FONCEA, M. \& MARCUELLO-SERVÓS, C. (2010): "Impacto económico de las cooperativas. La generación de empleo en las sociedades cooperativas y su relación con el PIB", CIRIEC-España, Revista de Economía Pública, Social y Cooperativa, 67, 23-44.

ESTRIN, S. \& JONES, D.C. (1992): "The viability of employee-owned firms: Evidence from France", Industrial \& Labor Relations Review, 45(2), 323. Retrieved from http://search.proquest.com/docview/236321868?accountid=17248

FERNÁNDEZ GUADAÑO, J. (2007): "Divergencias entre las normas internacionales de información financiera y las normas sobre aspectos contables de las sociedades cooperativas: Efectos sobre la solvencia financiera". Comunicación presentada al XX Congreso Anual de AEDEM, Decisiones Basadas en el Conocimiento y en el Papel Social de la Empresa, p. 61.

FERNÁNDEZ-FEIJÓO, B. \& CABALEIRO, M.J. (2007): "Clasificación del capital social de la sociedad cooperativa: Una visión crítica", CIRIEC-España, Revista de Economía Pública, Social y Cooperativa, 58, 7-29.

FURUBOTN, E.G. \& PEJOVICH, S. (1970): "Property rights and the behavior of the firm in a socialist state: The example of Yugoslavia", Journal of Economics, 30(3), 431-454.

GARCÍA-GUTIÉRREZ FERNÁNDEZ, C. (1986): "Estudio del régimen económico y de la contabilidad de la empresa cooperativa en relación con la ley 3/1987, de 2 de abril, general de cooperativas", REVESCO, Revista de Estudios Cooperativos, 54, 169-224.

GENOVART BALAGUER, J.I. (2012): Aspectos económico-contables de las cooperativas, Tesis Doctoral, Universidad de las Islas Baleares, Obtenido de http://global.tesisenxarxa.net/bitstream/handle/10803/111331/tjgb1de1.pdf?sequence=1

GÓMEZ APARICIO, P. \& MIRANDA GARCÍA, M. (2006): "Sobre el régimen económico y financiero particular de las sociedades cooperativas", REVESCO, Revista de Estudios Cooperativos, 90, 28-56.

HENK, T. (1982): "The performance of the Mondragon cooperatives in Spain". In: Participatory and Self-Managed Firms, Ed. DC Jones \& J.Svejnar. Lexington Books, 129-151.

HIND, A.M. (1994): "Cooperatives-under performers by nature? An exploratory analysis of cooperative and non-cooperative companies in the agri-business sector", Journal of Agricultural Economics, 45(2), 213-219. 
MARÍ VIDAL, S. (2006): "Efectos de la aplicación de la CINIIF 2 en las cooperativas. Un estudio empírico en dos cooperativas citrícolas de la comunidad valenciana a través del análisis económicofinanciero", REVESCO, Revista de Estudios Cooperativos, 89, 84-107.

MARTíN LÓPEZ, S., LEJARRIAGA PÉREZ DE LAS VACAS, G. \& ITURRIOZ DEL CAMPO, J. (2007): "Consideraciones sobre la naturaleza del capital social en las sociedades cooperativas de trabajo asociado", REVESCO, Revista de Estudios Cooperativos, 91, 93-119.

MCKINSEY (2012): Mckinsey on Cooperatives, Editor Michael Borruso.

MONZÓN CAMPOS, J. \& CHAVES ÁVILA, R. (2012): La economía social en la Unión Europea, Comité Económico y Social Europeo.

NOTTA, O. \& VLACHVEI, A. (2007): "Performance of cooperatives and investor-owned firms: The case of the Greek dairy industry". In Kostas Karantininis \& Jerker Nilsson: Vertical markets and cooperative hierarchies, Springer, Chapter 16, 275-285.

OBSERVATORIO VASCO DE ECONOMÍA SOCIAL (2012): Informe de Situación de la Economía Social Vasca 2011, Disponible en:http://www.gezki.ehu.es/p281 content/es/contenidos/organo/behatoki_info/es_behat_1/adjuntos/InformedeSituaci\%C3\%B3ndelaEconom\%C3\%ADaSocialVasca2011201 2.pdf

ORMAECHEA, J.M. (1991): La experiencia cooperativa de Mondragón, Grupo Cooperativo Mondragón.

POLO-GARRIDO, F. (2007): "Impactos de las normas internacionales de información financiera en el régimen económico de las sociedades cooperativas", CIRIEC-España, Revista de Economía Pública, Social y Cooperativa, 58, 83-108.

POZUELO CAMPILLO, J., CARMONA IBÁÑEZ, P. \& MARTÍNEZ VARGAS, J. (2012): "Las sociedades cooperativas y las sociedades capitalistas en la Comunidad Valenciana: análisis comparado de su estructura económica y financiera", CIRIEC-España, Revista de Economía Pública, Social y Cooperativa, 74, 117-147.

SERVER, R. \& LAJARA-CAMILLERI, N. (2012): "Comportamiento del modelo cooperativo ante la crisis", Revista AECA, 99, 74-78.

SOBOH, R., OUDE LANSINK, A. \& VAN DIJK, G. (2011): "Distinguishing dairy cooperatives from investor-owned firms in Europe using financial indicators", Agribusiness, 27(1), 34-46.

SOBOH, R., OUDE LANSINK, A. \& VAN DIJK, G. (2012): "Efficiency of cooperatives and investor owned firms revisited", Journal of Agricultural Economics, 63(1), 142-157.

STIGLITZ, D.J. (2009): "Moving beyond market fundamentalism to a more balanced economy", Annals of Public and Cooperative Economics, 80(3), 345-360.

SYRJÄ, P., SJÖGREN, H. \& TUOMINEN, P. (2012): "Financial performance and efficiency of consumer co-operatives and limited companies-Agency theoretical approach", Journal of Co-operative Accounting and Reporting, 1, 53-69. 
VANEK, J. (1977): The labor-managed economy: Essays, Cornell University Press.

VARGAS VASSEROT, C. (2007): "Los previsibles efectos de la NIC 32 en el sector cooperativo", REVESCO, Revista de Estudios Cooperativos, 91, 120-159.

VARGAS VASSEROT, C. (2011): "Aportaciones exigibles o no exigibles: Ésa es la cuestión", CIRIECEspaña, Revista Jurídica de Economía Social y Cooperativa, 22, 75-119.

WHYTE, W.F. \& WHYTE, K.K. (1991): Making Mondragon: The growth and dynamics of the worker cooperative complex, Cornell University Press.

ZEVI, A., ZANOTTI, A., SOULAGE, F. \& ZELAIA, A. (2011): Más allá de la crisis: Cooperativas, trabajo, finanzas. Generación de riqueza para el largo plazo, Brussels, Belgium: Publicaciones de CECOP.

ZUBIAURRE ARTOLA, M.Á. (2004): "Sociedades cooperativas. Aspectos contables singulares", Cuadernos de Gestión, 4(2), 47-62.

ZUBIAURRE, M.Á., ANDICOECHEA, L. \& SAITUA, A. (2015): "Aplicación de la orden EHA/3360/2010 sobre aspectos contables de las sociedades cooperativas: efectos en los fondos propios de las cooperativas de la Comunidad Autónoma del País Vasco", REVESCO, Revista de Estudios Cooperativos, 118, 180-201.

\section{Legislación:}

Ley 4/1993, de 24 de junio, de Cooperativas de Euskadi. BOE n 35, de 10 de febrero de 2012. http://www.boe.es/boe/dias/2012/02/10/pdfs/BOE-A-2012-2011.pdf

Orden EHA/3360/2010, de 21 de Diciembre, por la que se aprueban las Normas sobre los aspectos contables de las sociedades cooperativas. BOE $n^{\circ} 316$ de 29 de Diciembre de 2010. https://www.boe.es/buscar/doc.php?id=BOE-A-2010-20034

Real Decreto 1514/2007, de 16 de noviembre, por el que se aprueba el Plan General de Contabilidad. BOE ${ }^{0} 278$, de 20 de noviembre de 2007. http://www.boe.es/diario_boe/txt.php?id=BOE-A-200719884 
ANEXO: Pruebas de Normalidad

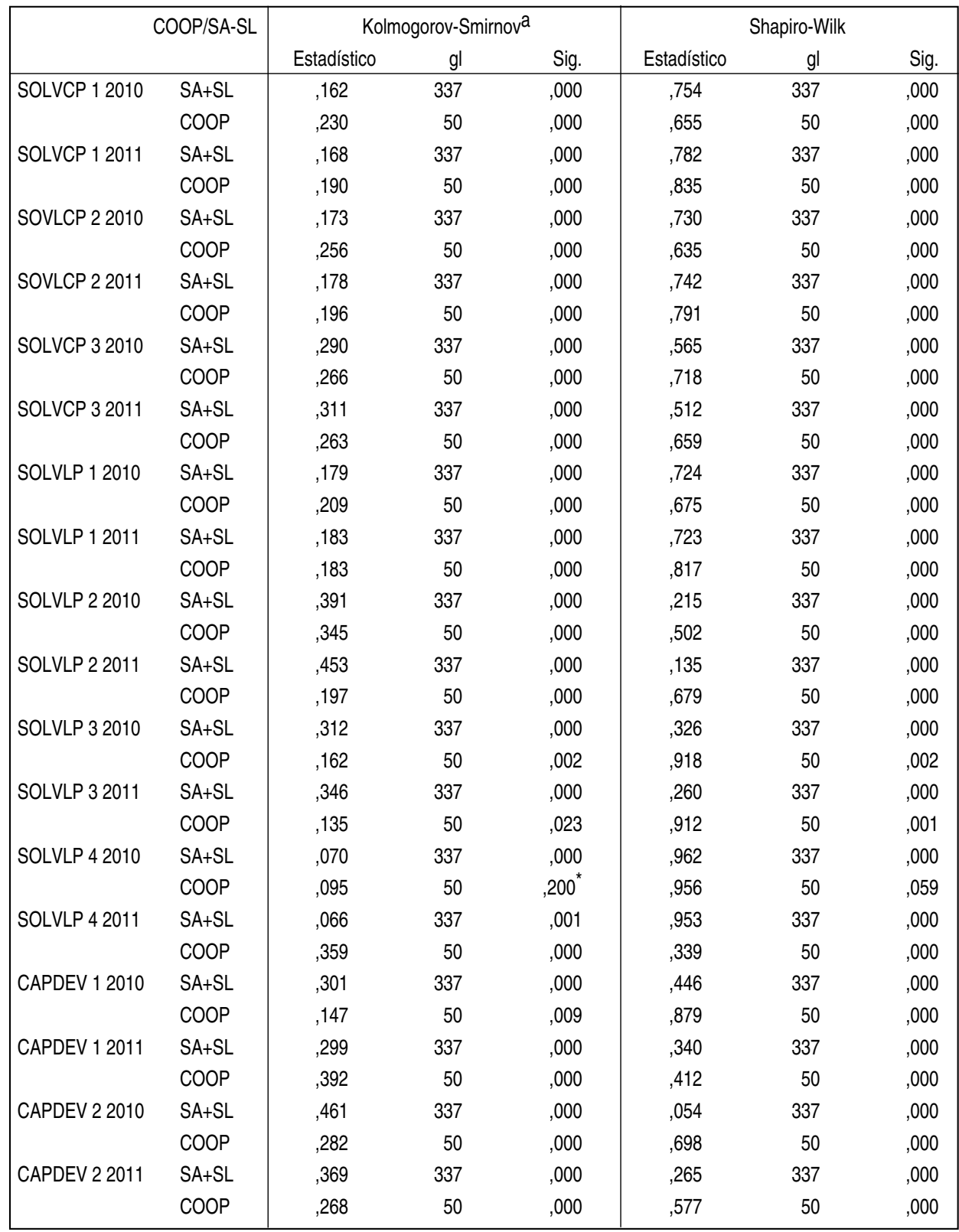




\begin{tabular}{|c|c|c|c|c|c|c|c|}
\hline \multirow[t]{2}{*}{ RENTB 12010} & $\mathrm{SA}+\mathrm{SL}$ & 181 & 337 &, 000 &, 521 & 337 &, 000 \\
\hline & $\mathrm{COOP}$ & ,184 & 50 &, 000 & ,908 & 50 & ,001 \\
\hline \multirow[t]{2}{*}{ RENTB 12011} & $\mathrm{SA}+\mathrm{SL}$ & , 178 & 337 &, 000 & ,678 & 337 &, 000 \\
\hline & COOP & ,176 & 50 & ,001 & 818 & 50 &, 000 \\
\hline \multirow[t]{2}{*}{ RENTB 22010} & $\mathrm{SA}+\mathrm{SL}$ & ,177 & 337 &, 000 & ,671 & 337 &, 000 \\
\hline & $\mathrm{COOP}$ & ,161 & 50 &, 002 & ,907 & 50 &, 001 \\
\hline \multirow[t]{2}{*}{ RENTB 22011} & $\mathrm{SA}+\mathrm{SL}$ & ,345 & 337 &, 000 & , 196 & 337 &, 000 \\
\hline & $\mathrm{COOP}$ & ,213 & 50 &, 000 & ,791 & 50 &, 000 \\
\hline \multirow[t]{2}{*}{ RENTB 32010} & $\mathrm{SA}+\mathrm{SL}$ & 183 & 337 &, 000 & ,537 & 337 &, 000 \\
\hline & $\mathrm{COOP}$ & ,106 & 50 &, $200^{*}$ & 947 & 50 &, 025 \\
\hline \multirow[t]{2}{*}{ RENTB 32011} & $\mathrm{SA}+\mathrm{SL}$ & ,180 & 337 &, 000 & ,685 & 337 &, 000 \\
\hline & $\mathrm{COOP}$ & , 160 & 50 & ,003 & 826 & 50 &, 000 \\
\hline \multirow[t]{2}{*}{ RENTB 42010} & $\mathrm{SA}+\mathrm{SL}$ & ,176 & 337 &, 000 & ,693 & 337 &, 000 \\
\hline & $\mathrm{COOP}$ & ,136 & 50 & ,022 & ,917 & 50 &, 002 \\
\hline \multirow[t]{2}{*}{ RENTB 42011} & $\mathrm{SA}+\mathrm{SL}$ & ,338 & 337 &, 000 & 203 & 337 &, 000 \\
\hline & COOP & 277 & 50 &, 000 & ,725 & 50 &, 000 \\
\hline \multirow[t]{2}{*}{ RENTB 52010} & $\mathrm{SA}+\mathrm{SL}$ & ,207 & 337 &, 000 & ,473 & 337 &, 000 \\
\hline & $\mathrm{COOP}$ & ,106 & 50 & ,200* & ,953 & 50 &, 045 \\
\hline \multirow[t]{2}{*}{ RENTB 52011} & $\mathrm{SA}+\mathrm{SL}$ & ,201 & 337 &, 000 & ,629 & 337 &, 000 \\
\hline & $\mathrm{COOP}$ & , 158 & 50 &, 003 & 811 & 50 &, 000 \\
\hline \multirow[t]{2}{*}{ RENTB 62010} & $\mathrm{SA}+\mathrm{SL}$ & ,185 & 337 &, 000 & 695 & 337 &, 000 \\
\hline & $\mathrm{COOP}$ & ,131 & 50 & ,031 & ,917 & 50 & ,002 \\
\hline \multirow[t]{2}{*}{ RENTB 62011} & $\mathrm{SA}+\mathrm{SL}$ & ,351 & 337 &, 000 & , 187 & 337 &, 000 \\
\hline & $\mathrm{COOP}$ & 275 & 50 &, 000 & ,714 & 50 &, 000 \\
\hline \multirow[t]{2}{*}{ RENTB 72010} & $\mathrm{SA}+\mathrm{SL}$ & ,177 & 337 &, 000 &, 543 & 337 &, 000 \\
\hline & $\mathrm{COOP}$ &, 067 & 50 & ,200* & 940 & 50 &, 013 \\
\hline \multirow[t]{2}{*}{ RENTB 72011} & $\mathrm{SA}+\mathrm{SL}$ & ,121 & 337 &, 000 & ,760 & 337 &, 000 \\
\hline & $\mathrm{COOP}$ & ,355 & 50 &, 000 & ,363 & 50 &, 000 \\
\hline \multirow[t]{2}{*}{ RENTB 82010} & $S A+S L$ & ,347 & 337 &, 000 & ,214 & 337 &, 000 \\
\hline & $\mathrm{COOP}$ & ,155 & 50 &, 004 & ,888 & 50 &, 000 \\
\hline \multirow[t]{2}{*}{ RENTB 82011} & $S A+S L$ & ,438 & 337 &, 000 & ,117 & 337 &, 000 \\
\hline & $\mathrm{COOP}$ & ,129 & 50 &, 036 & ,908 & 50 &, 001 \\
\hline \multirow[t]{2}{*}{ RENTB 92010} & $\mathrm{SA}+\mathrm{SL}$ & ,412 & 337 &, 000 & ,157 & 337 &, 000 \\
\hline & $\mathrm{COOP}$ & ,286 & 50 &, 000 & ,414 & 50 &, 000 \\
\hline \multirow[t]{2}{*}{ RENTB 92011} & $\mathrm{SA}+\mathrm{SL}$ & ,366 & 337 &, 000 & 273 & 337 &, 000 \\
\hline & $\mathrm{COOP}$ & ,399 & 50 &, 000 & ,305 & 50 &, 000 \\
\hline \multirow[t]{2}{*}{ RENTB 102010} & $\mathrm{SA}+\mathrm{SL}$ &, 418 & 337 &, 000 & ,207 & 337 &, 000 \\
\hline & $\mathrm{COOP}$ & ,335 & 50 &, 000 &, 511 & 50 &, 000 \\
\hline \multirow[t]{2}{*}{ RENTB 102011} & $\mathrm{SA}+\mathrm{SL}$ & ,470 & 337 &, 000 & 109 & 337 &, 000 \\
\hline & $\mathrm{COOP}$ & ,312 & 50 &, 000 &, 578 & 50 &, 000 \\
\hline
\end{tabular}




\begin{tabular}{|c|c|c|c|c|c|c|c|}
\hline \multirow[t]{2}{*}{ RENTB 112010} & $\mathrm{SA}+\mathrm{SL}$ & 397 & 337 &, 000 & , 150 & 337 &, 000 \\
\hline & $\mathrm{COOP}$ & 277 & 50 &, 000 &, 592 & 50 &, 000 \\
\hline \multirow[t]{2}{*}{ RENTB 112011} & $\mathrm{SA}+\mathrm{SL}$ & ,445 & 337 &, 000 & 106 & 337 &, 000 \\
\hline & COOP & 169 & 50 &, 001 & ,870 & 50 &, 000 \\
\hline \multirow[t]{2}{*}{ VAÑAD 12010} & $\mathrm{SA}+\mathrm{SL}$ & , 199 & 337 &, 000 & ,648 & 337 &, 000 \\
\hline & $\mathrm{COOP}$ & ,112 & 50 & ,161 & ,922 & 50 &, 003 \\
\hline \multirow[t]{2}{*}{ VAÑAD 12011} & $S A+S L$ & 174 & 337 &, 000 & ,639 & 337 &, 000 \\
\hline & COOP & ,116 & 50 & ,091 & 910 & 50 &, 001 \\
\hline \multirow[t]{2}{*}{ VAÑAD 22010} & $\mathrm{SA}+\mathrm{SL}$ & , 149 & 337 &, 000 &, 742 & 337 &, 000 \\
\hline & $\mathrm{COOP}$ & 184 & 50 &, 000 &, 860 & 50 &, 000 \\
\hline \multirow[t]{2}{*}{ VAÑAD 22011} & $\mathrm{SA}+\mathrm{SL}$ & ,357 & 337 &, 000 & ,116 & 337 &, 000 \\
\hline & $\mathrm{COOP}$ & ,278 & 50 &, 000 &, 555 & 50 &, 000 \\
\hline \multirow[t]{2}{*}{ VAÑAD 32010} & $\mathrm{SA}+\mathrm{SL}$ & 174 & 337 &, 000 & ,676 & 337 &, 000 \\
\hline & $\mathrm{COOP}$ & , 181 & 50 &, 000 & ,696 & 50 &, 000 \\
\hline \multirow[t]{2}{*}{ VAÑAD 32011} & $\mathrm{SA}+\mathrm{SL}$ & ,121 & 337 &, 000 & ,817 & 337 &, 000 \\
\hline & $\mathrm{COOP}$ & ,172 & 50 &, 001 &, 759 & 50 &, 000 \\
\hline \multirow[t]{2}{*}{ PRODT 12010} & $\mathrm{SA}+\mathrm{SL}$ & ,419 & 337 &, 000 &, 076 & 337 &, 000 \\
\hline & $\mathrm{COOP}$ & ,112 & 50 & ,154 & ,973 & 50 & ,302 \\
\hline \multirow[t]{2}{*}{ PRODT 12011} & $\mathrm{SA}+\mathrm{SL}$ & 333 & 337 &, 000 & ,255 & 337 &, 000 \\
\hline & $\mathrm{COOP}$ & 161 & 50 &, 002 & ,765 & 50 &, 000 \\
\hline \multirow[t]{2}{*}{ PRODT 22010} & $\mathrm{SA}+\mathrm{SL}$ & ,131 & 337 &, 000 & ,867 & 337 &, 000 \\
\hline & $\mathrm{COOP}$ & ,179 & 50 &, 000 & ,782 & 50 &, 000 \\
\hline \multirow[t]{2}{*}{ PRODT 22011} & $\mathrm{SA}+\mathrm{SL}$ & ,303 & 337 &, 000 & ,195 & 337 &, 000 \\
\hline & $\mathrm{COOP}$ & 264 & 50 &, 000 &, 577 & 50 &, 000 \\
\hline \multirow[t]{2}{*}{ PRODT 32010} & $\mathrm{SA}+\mathrm{SL}$ & ,231 & 337 &, 000 &, 586 & 337 &, 000 \\
\hline & $\mathrm{COOP}$ & 232 & 50 &, 000 & ,671 & 50 &, 000 \\
\hline \multirow[t]{2}{*}{ PRODT 32011} & $\mathrm{SA}+\mathrm{SL}$ & 217 & 337 &, 000 &, 554 & 337 &, 000 \\
\hline & $\mathrm{COOP}$ & ,359 & 50 &, 000 & ,318 & 50 &, 000 \\
\hline \multirow[t]{2}{*}{ PRODT 42010} & $\mathrm{SA}+\mathrm{SL}$ & 201 & 337 &, 000 &, 721 & 337 &, 000 \\
\hline & $\mathrm{COOP}$ & ,209 & 50 &, 000 &, 745 & 50 &, 000 \\
\hline \multirow[t]{2}{*}{ PRODT 42011} & $S A+S L$ & , 166 & 337 &, 000 & ,791 & 337 &, 000 \\
\hline & COOP & 138 & 50 & ,018 & ,893 & 50 &, 000 \\
\hline
\end{tabular}

*. Esto es un límite inferior de la significación verdadera.

a. Corrección de significación de Lilliefors. 